\title{
Sensitivity of wavepackets in jets to non-linear effects: the role of the critical layer
}

\author{
Gilles Tissot; $\quad$ Mengqi Zhang†’ Francisco C. Lajús Jr.‡ \\ André V.G. Cavalieri\$ Peter Jordan " and Tim Colonius॥
}

\begin{abstract}
Linear instability waves, wavepackets, are key building blocks for the jet-noise problem. It has been shown in previous work that linear models correctly predict the evolution of axisymmetric wavepackets up to the end of the potential core. Beyond this station linear models fail to predict single-point statistics; they fail more broadly in the prediction of two-point properties such as coherence; and their underprediction of the radiated noise is believed to be associated with these errors. Non-linearity is the likely missing piece. But how might it be incorporated? What are the essential underlying mechanisms? Might it be amenable to a reduced-order modelling methodology? The work described in this paper is concerned with these questions. The non-linear interactions are considered as an "external" harmonic forcing of the standard linear model; the forcing can be viewed as comprising those Fourier components of the non-linear term of the Navier-Stokes equations which are most amplified by the linear wavepackets.

This modelling framework is explored using three complementary problems in which we try to understand the relationship between "external" forcing, linear system and flow response. The response of an incompressible, two-dimensional, locally parallel, shear-flow to direct, spatially localised, harmonic forcing is first considered. A resolvant analysis is then performed, again in a locally parallel context, both for the incompressible, 2D problem and for a compressible axisymmetric shear-flow where the mean flow is taken from experiments. Finally, in order to incorporate the slow axial variation of the real jet, a novel approach is considered where 4D-Var data assimilation is applied using experimental data and the Parabolised Stability Equations (PSE-4D-Var). The objective of this third, data-driven, approach is to search for an optimal forcing that might improve the match between wavepaket solutions and measurements.

In all of the problems considered the critical layer, where the phase speed of the wave is equal to the local mean velocity, is found to be relevant. It is at this point that the sensitivity of the linear waves to non-linearity is greatest. In the $2 \mathrm{D}$, incompressible, problem the largest response is produced when the flow is forced in the vicinity of the critical layer. The resolvant analyses show optimal forcing modes that peak on the critical layer and the optimal response modes have a critical-layer structure. The PSE-4D-Var approach shows highest sensitivity near the critical layer. Furthermore, the structure of the forced perturbations are tilted in a manner that suggests an Orr-like mechanism.

The ensemble of results suggest that the critical layer may play a central role in the modelling of wavepackets in subsonic turbulent jets, and indeed may be the key to remedying the deficiencies evoked above.
\end{abstract}

\footnotetext{
*Post-Doc, Instituto Tecnológico, Divisão de Engenharia Aeronáutica, Praça Mal. Eduardo Gomes, 50, Vila da Acácias, 12228-900, São José dos Campos - SP, Brazil

${ }^{\dagger}$ PhD Student, Institut Pprime, CNRS-University of Poitiers-ENSMA, Poitiers, France.

¥Phd Student, Depart. de Eng. Mecânica, Universidade Federal de Santa Catarina, Fpolis, SC, Brazil

$\S$ Assistant Professor, Instituto Tecnológico, Divisão de Engenharia Aeronáutica, Praça Mal. Eduardo Gomes, 50, Vila da Acácias, 12228-900, São José dos Campos - SP, Brazil

IResearch Scientist, Institut Pprime, CNRS-University of Poitiers-ENSMA, Poitiers, France.

" Professor, Associate Fellow AIAA, Mechanical Engineering, California Institute of Technology, Pasadena, CA 91125, USA

Copyright (c) 2012 by the authors. Published by the American Institute of Aeronautics and Astronautics, Inc. with permission.
} 


\section{Introduction}

Numerous studies support the idea that wavepackets, obtained as linear stability solutions with the mean velocity field as a base flow, dominate at least the low-angle sound radiation ${ }^{1}$ of subsonic turbulent jets. The structure of the sound field is consistent with a wavepacket source at low emission angles, ${ }^{2}$ and wavepackets educed from data in the hydrodynamic region agree closely with linear theory in regions upstream of the end of the potential core and over a broad range of Strouhal numbers. ${ }^{3}$ The agreement is, however, poor in all regions at Strouhal numbers below $\mathrm{St}=0.3$, and downstream of the end of the potential core for all Strouhal numbers (see figure 1). Moreover, noise predicted by such linear wavepacket models is orders of magnitude lower than experimental results. ${ }^{4}$ Two-point coherence is another quantity that the linear models cannot model correctly. ${ }^{5-8}$ These discrepancies are all believed to be due to a dynamic trait of wavepackets, related to non-linearities, that we have given the term jitter, and that has been shown to be important by a number of studies for obtaining quantitatively accurate noise prediction. ${ }^{8-10}$

A simplified dynamical model, that attempts to account for this behavior by implicitly including nonlinearity by allowing wavepackets to evolve on an unsteady base flow, was recently tested by Zhang et al. ${ }^{4}$ and by Jordan et al. ${ }^{8}$ The model produced jittering wavepackets with considerably enhanced sound radiation, but it was found that the noise increase was not sufficient to explain the observed levels. It appears, therefore, that non-linearity must be accounted for differently. In this work, we consider the non-linearity, which can be rigourously expressed as generalised Reynolds stresses, as an "external" forcing ${ }^{\mathrm{a}}$, and we will focus on its influence on the flow instability. Such a framework was first envisaged by Landahl. ${ }^{11}$ Two ways of modelling these external forcings are possible, stochastic or deterministic. Concerning the former, a related study considers the issue from the perspective of balanced modes between the hydrodynamic non-linearities and the acoustic field of a higher Mach-number jet. ${ }^{12}$ We consider the latter, assuming that the forcing is deterministic in spectral space. The objective in this context is then to study relationship between external forcing and flow response, these being and constrained by the linearised model.

In shear flows, a region, called the critical layer, is known ${ }^{13-16}$ to be highly sensitive to external forcing. At the critical layer, the phase speed of a propagating wave is equal to the local speed of the base flow, and this gives rise to a singularity in the inviscid shear-flow problem. This singularity is usually overcome by considering either viscous effects or nonlinearity ${ }^{17}$ (for high Reynolds number jets, the non-linearity is $a$ priori the dominant term), both having the potential to produce significant effects even when present at low amplitudes. In this work, we study the response of shear flow to external forcing and we find the role of the critical layer to be important in describing the effects of non-linearity on wavepacket evolution.

Three problems, of varying degrees of complexity, are considered. The first approach addresses the problem directly by probing a 2D incompressible shear flow, forced by time-periodic disturbances localised in space, and studying the behavior of the response. This allows us to explore the sensitivity of different regions of the shear flow to external forcing, focusing in particular on how the presence of both critical layer and inflection point may affect the sensitivity of the flow response to nonlinearities. We compute the response to a Dirac forcing using canonical profiles: a Couette flow profile and a hyperbolic tangent profile.

The second approach, resolvent analysis, involves examination of the flow system as a linear operator. Optimal harmonic forcing and associated responses are obtained by performing singular value decomposition of the resolvent of the linearised Navier-Stokes operator. This non-modal stability technique has been applied by Sharma \& McKeon ${ }^{18}$ to model coherent structures in a turbulent pipe flow and has been put in perspective with the critical-layer mechanism in McKeon \& Sharma. ${ }^{19}$ Concerning amplifier flows ${ }^{\mathrm{b}}$, a backward facing step has been studied in a global setting by Dergham et al.,${ }^{20}$ who showed the link between harmonic and stochastic forcing analyses. Garnaud et al. ${ }^{21}$ and Nichols et al. ${ }^{22}$ also considered a global approach for exploring the amplifier character of jets. In our study, we adopt a locally parallel framework for cylindrical compressible flows as a complementary approach. Indeed, beyond the computational advantages compared to global analysis, it allows additional interpretations of physical phenomena by focusing on localised regions of the flow. ${ }^{23}$ Specifically, optimal response modes (forced wavepackets) and optimal forcing modes (the modes through which non-linear interactions force wavepackets) are computed in different regions of the jet and compared with linear wavepacket results and experimental data for a $M=0.4$ jet which has been examined in several previous studies. ${ }^{2,3,24,25}$

\footnotetext{
aThe term "external" forcing is used in this paper because the tools used here come from input-output frameworks. However, this forcing represents non-linear effects, which are physically "internal", unlike an actuation for instance.

${ }^{\mathrm{b}}$ Globally stable flows, which amplify incoming disturbances in space.
} 
The third approach, the 4D data assimilation (4D-Var), solves the input-output problem in an inverse manner by searching for the optimal forcing profile giving rise to improved agreement between flow response and measurements, thus allowing us to explore the missing pieces in the linear model for an accurate description of wavepackets observed in the data. The 4D-Var technique is data-driven in nature and has been used extensively in weather forecast ${ }^{26}$ to combine different sources of inhomogeneous informations: a simplified model, noisy and sparse observations and an a priori knowledge of the solution. Besides, it has also evolved as a powerful flow control technique. ${ }^{27}$ We introduce a novel use of this method for wavepacket modelling, in a slowly-diverging base flow using the parabolised stability equations (PSE). Specifically, we wish to identify the non-linear terms, not accounted for the linear model. Interpretation of the results of this approach is considerably aided by the results of the first two approaches.

The paper is organised as follows. In section II, we introduce the viewpoint that consists in considering the harmonic external forcing as Fourier components of the nonlinear terms in the Navier-Stokes equations. In section III, we formulate the mathematical problem for each of the approaches listed above and show results. Finally in section IV, we conclude the paper with a discussion of the results and some perspectives.

\section{Non-linear effect as external forcing}

The linear growth of Kelvin-Helmoltz (K-H) instabilities is well predicted by linear models, ${ }^{1}$ such as PSE, for a wide range ${ }^{28}$ of frequencies until approximately the end of the potential core. We focus on the axisymmetric mode $(m=0)$, which is the most acoustically efficient. ${ }^{3}$ Figure 1 shows the comparison between hot-wire measurements and PSE on the centerline of a $M=0.4$ jet from $\mathrm{St}=0.1$ to $\mathrm{St}=0.9$. Beyond the potential core, due to the spreading of the jet, linear model predicts a decay. However, experiments ${ }^{3}$ show an increase of the amplitude of the axisymmetric wavepacket on the centerline ${ }^{\mathrm{c}}$. In order to correctly describe the wavepacket throughout the flow, the linear model is no longer sufficient and non-linearities must be incorporated in some way. We have chosen in this paper to consider non-linear effects on the wavepacket as a harmonic forcing.

For the flow variable $\boldsymbol{q}_{\phi}=(\boldsymbol{u}, T, \rho)$, containing the $N_{c}$ components of velocity, temperature and density, we consider the Reynolds decomposition $\boldsymbol{q}_{\phi}(\boldsymbol{x}, t)=\overline{\boldsymbol{q}}(\boldsymbol{x})+\boldsymbol{q}^{\prime}(\boldsymbol{x}, t)$, where $\overline{\boldsymbol{q}}(\boldsymbol{x})$ is the mean flow and $\boldsymbol{q}^{\prime}(\boldsymbol{x}, t)$ is the fluctuation. In cylindrical coordinates, the space variable $\boldsymbol{x}=(x, r, \theta)$ is expressed by its axial, radial and azimuthal coordinates. For a jet, using homogeneity in the azimuthal direction, we decompose the fluctuation in Fourier modes

$$
\boldsymbol{q}^{\prime}(x, r, \theta, t)=\sum_{\omega} \sum_{m} \widetilde{\boldsymbol{q}}_{\omega, m}(x, r) e^{i(m \theta-\omega t)} .
$$

Introducing decomposition (1) in the Navier-Stokes (NS) equations, we obtain in operator notation

$$
\mathcal{L}_{\overline{\boldsymbol{q}}, \omega, m} \widetilde{\boldsymbol{q}}_{\omega, m}=\mathcal{N}_{\overline{\boldsymbol{q}}, \omega, m}\left(\boldsymbol{q}^{\prime}\right),
$$

where $\mathcal{L}_{\overline{\boldsymbol{q}}, \omega, m}$ is the linearised NS operator around the base flow $\overline{\boldsymbol{q}}$ and $\mathcal{N}_{\overline{\boldsymbol{q}}, \omega, m}\left(\boldsymbol{q}^{\prime}\right)$, neglected in the linear stability analysis, represents the Fourier component $(\omega, m)$ of the nonlinear terms. In linear stability theory for jet flow, ${ }^{31}$ one usually finds $\widetilde{\boldsymbol{q}}_{\omega, m}$, the K-H mode, as a highly unstable mode giving rise to an exponential perturbation growth in the near-nozzle region; this corresponds to the homogeneous linear system $\mathcal{L}_{\overline{\boldsymbol{q}}, \omega, m} \widetilde{\boldsymbol{q}}_{\omega, m}=0$. The nonlinear terms $\mathcal{N}_{\overline{\boldsymbol{q}}, \omega, m}\left(\boldsymbol{q}^{\prime}\right)$ becomes significant when these disturbance manifests large amplitudes or if the linear operator is highly sensitive to this forcing. Because the non-linear term depends on $\boldsymbol{q}^{\prime}$, it is the result of a convolution of all $\widetilde{\boldsymbol{q}}_{\omega, m}$, for all $(\omega, m)$ modes, as illustrated figure 2 .

Sections III.B and III.C consider locally parallel frameworks for 2D incompressible ${ }^{\mathrm{d}}$ and axisymmetric compressible shear flows, respectively. These formulations permit Fourier modes in the streamwise direction, and so the linearised model is written as $\mathcal{L}_{\bar{q}, \alpha, \omega, m}$, where $\alpha$ is the axial wavenumber of the considered perturbation. This locally parallel flow assumption will then be relaxed in the PSE-4D-Var approach section III.D.

Of interest in this paper is the characterisation of the operator $\mathcal{L}_{\bar{q}, \omega, m}$ in terms of the response with respect to an external forcing, $B \widetilde{\boldsymbol{f}}_{\omega, m}$, playing the role of the nonlinearities $\mathcal{N}_{\overline{\boldsymbol{q}}, \omega, m}\left(\boldsymbol{q}^{\prime}\right)$. As pictured in figure 3, we isolate the linearised operator for one frequency-wavenumber combination, and we characterise

\footnotetext{
${ }^{\mathrm{c}}$ Note that the near-field pressure, for instance, presents a downstream decay. ${ }^{7,29}$

${ }^{\mathrm{d}}$ In that case the azimuthal index $m$ is dropped.
} 

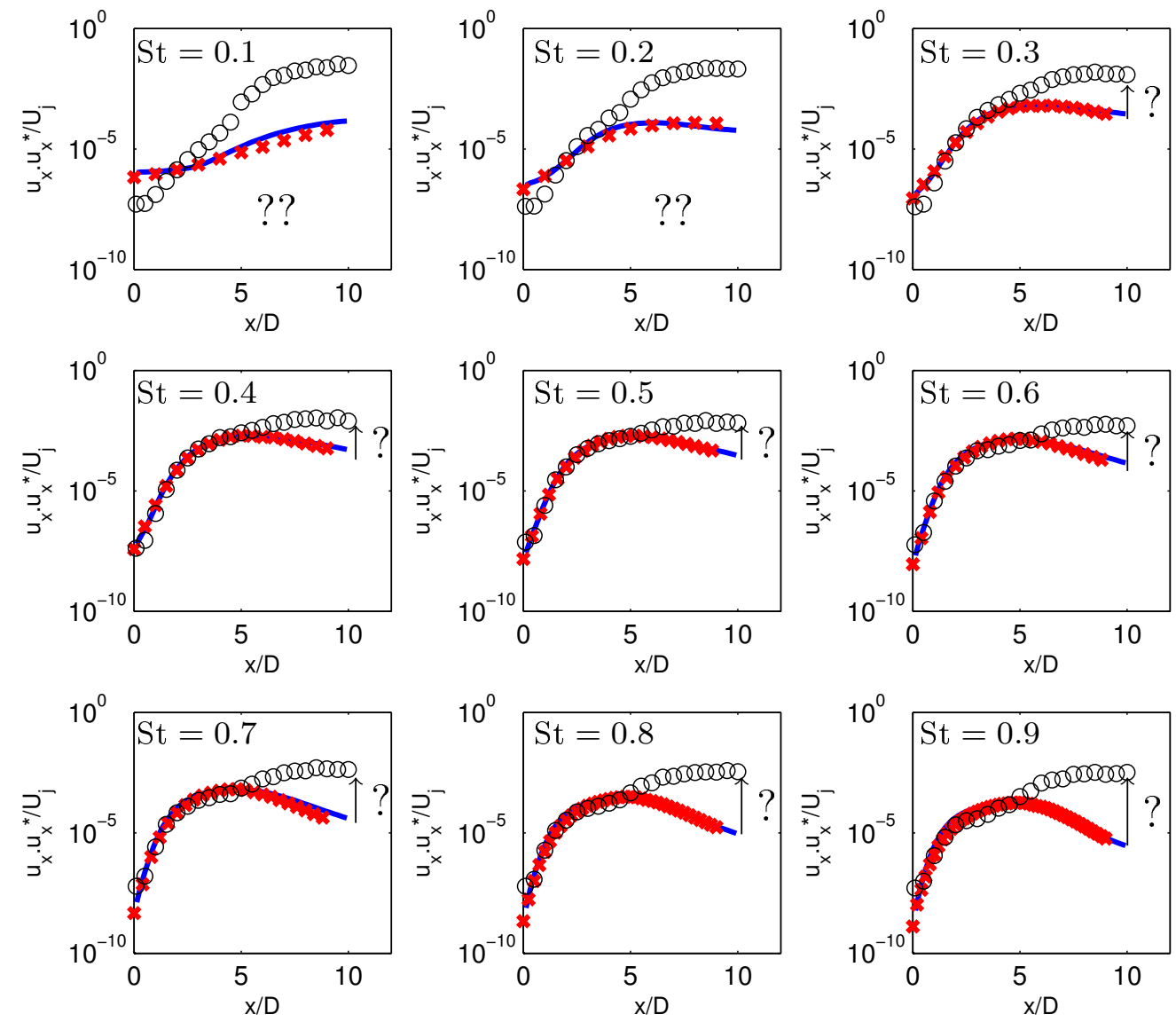

Figure 1. Measured streamwise velocity fluctuation on the jet centerline (black circles) as a function of axial position, compared with the PSE results of Cavalieri et al. ${ }^{3}$ (in red) and the results of the present PSE code (in blue). Figure from Piantanida et al. ${ }^{30}$

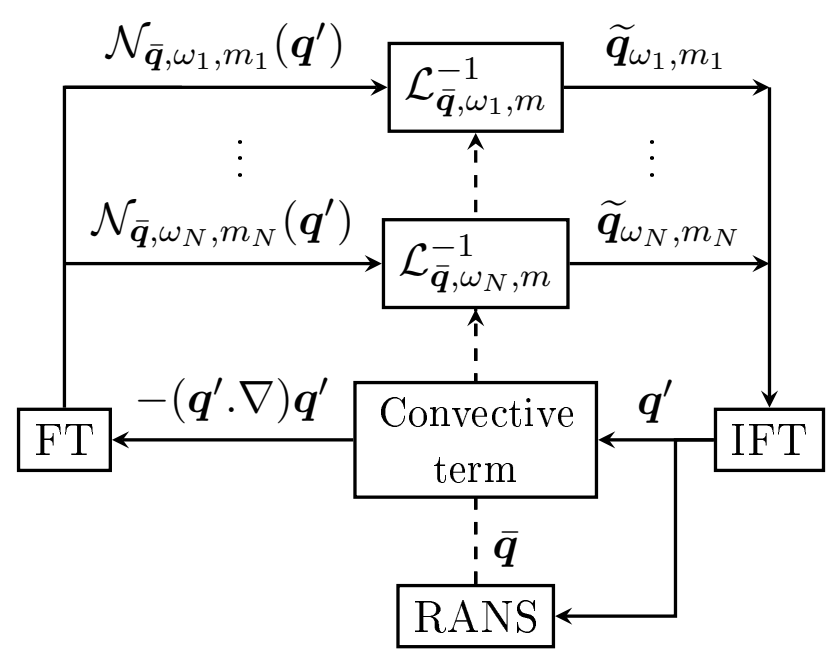

Figure 2. Schematic representation of the full non-linear system in the point of view of the response of the linearised operator to a non-linear forcing. Inspired by Moarref et al. ${ }^{32}$ "FT" refers for the Fourier transform (1) and "IFT" refers for the inverse Fourier transform. "RANS" holds for the Reynolds-Averaged Navier-Stokes equations. 
its response to a forcing. For further consistency, an input matrix $B$ is introduced symbolising that the forcing is restricted to a subspace, spanned by the image of $B$. It can restrict, for instance, the forcing to act only on the momentum equation. Similarly, an output matrix, $H$, is introduced to span an output observation space. It will be used explicitly in section III.C for the resolvent analysis and in section III.D for taking data into account in a data-assimilation procedure.

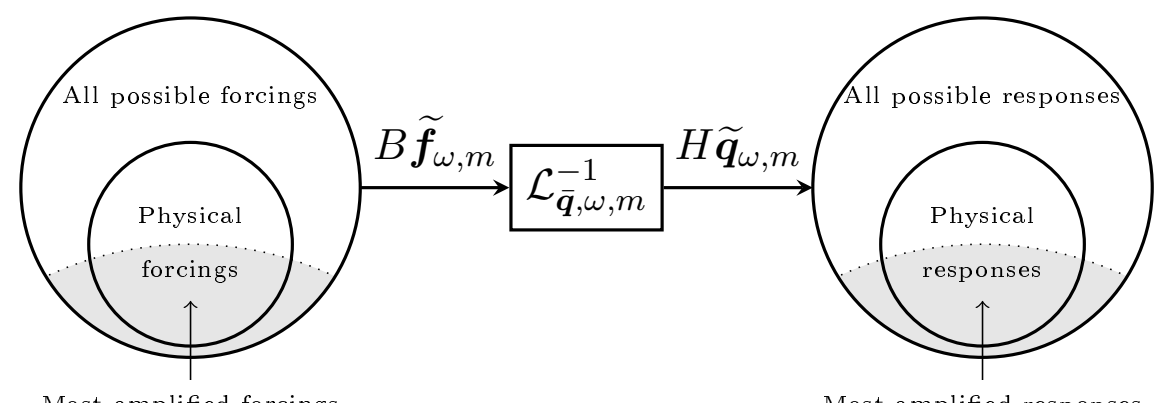

Figure 3. Schematic representation of the study of the input/output behavior of the linearised operator.

In a reduced-order modelling perspective, as represented in figure 3, the goal is to shrink the space of all possible forcings to only those which will be highly amplified by the flow. Consideration of how the non-linear term in the real jet exploits these mechanisms will be the subject of a later effort.

\section{Flow response to external forcing}

In this section, we present the mathematical formulations and results of the different methods considered for exploring flow response to external harmonic forcing. The critical-layer concept is first presented (section III.A) in order to highlight and discuss the associated physical mechanisms. The three numerical approaches, schematically represented in figure 4, are then employed to investigate in detail the input-output behaviour of the three flows considered. The approaches are hierarchised according to the complexity of the flow configurations considered. In the first (section III.B), the direct forcing, we consider a bi-dimensional, incompressible, parallel flow in Cartesian coordinates. We aim to demonstrate by this method the general characteristics of the critical layer in a simple case. After characterising the response to a direct forcing, we consider the optimal forcing and response associated with the flow. In the second approach (section III.C), the resolvent analysis, optimal forcing/response modes are determined in the same incompressible flow. Then, still in a locally parallel framework, an axisymmetric compressible jet flow is studied and its optimal response is compared to measurements. In the third approach (section III.D), PSE-4D-Var, the flow configuration is more realistic (slowly diverging thanks to the PSE formulation) and the method is driven by measurements. The results of this method show which regions in the jet are most sensitive to external forcing. This is particularly interesting as it can provide information regarding the missing piece of existing linear wavepacket models.

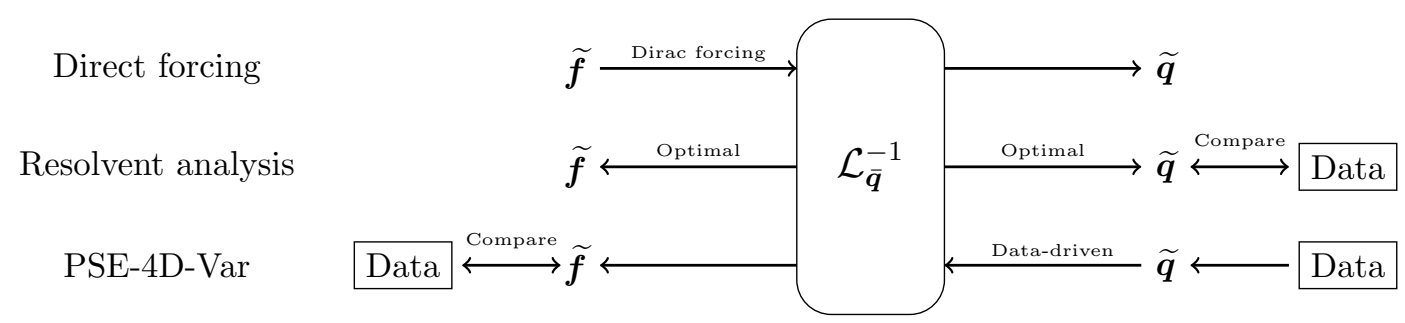

Figure 4. Three different approaches adopted in the paper. $\widetilde{q}$ represents the flow response, $\tilde{f}$ external forcing, $\mathcal{L}_{\bar{q}}^{-1}$ the resolvent of linear operator under the base flow $\overline{\boldsymbol{q}}$. The indices $\cdot \overline{\boldsymbol{q}}, \alpha, \omega, m$ are here dropped. 


\section{III.A. Critical layer}

Critical layers arise in inviscid, locally parallel, shear flows as a mathematical singularity of the linearised Euler equations at points where the phase velocity is equal to the local fluid velocity $\left(c=\bar{U}\left(y_{c}\right)\right)$. This can be illustrated by considering the Rayleigh equation, here expressed in terms of the stream function $\phi(x, y, t)=\widetilde{\phi}(y) e^{i \alpha(x-c t)}$,

$$
(\bar{U}-c)\left(\frac{\partial^{2}}{\partial y^{2}}-\alpha^{2}\right) \widetilde{\phi}-\frac{\partial^{2} \bar{U}}{\partial y^{2}} \widetilde{\phi}=0
$$

Solutions in the neighbourhood of this regular singularity can be investigated by application of the Frobenius method. ${ }^{13}$ Two linearly independent solutions of the homogeneous problem, in the vicinity of $y_{c}$, are shown to have the forms:

$$
\begin{aligned}
& \widetilde{\phi}_{A}=\left(y-y_{c}\right)+\left(\frac{\bar{U}_{c}^{\prime \prime}}{\bar{U}_{c}^{\prime}}\right) \frac{\left(y-y_{c}\right)^{2}}{2}+\ldots \\
& \widetilde{\phi}_{B}=1+\ldots+\left(\frac{\bar{U}_{c}^{\prime \prime}}{\bar{U}_{c}^{\prime}}\right) \phi_{A} \log \left(y-y_{c}\right)+\ldots
\end{aligned}
$$

where a log-singularity appears in $\phi_{B}$. A subsequent treatment of this logarithmic term ${ }^{13,33}$ reveals a $-\pi$ phase jump in the solution that causes a discontinuity in the stream function and then in the streamwise velocity along the transversal direction. ${ }^{13-15,33}$ So, this solution of the homogeneous problem comprises a dip in the transverse profile of the axial fluctuation magnitude: the signature of vortex-like structure.

Alternatively, the singularity can be observed in the linear operator of the system, by considering its response to an external force. In principle, there are two ways to get a large response. ${ }^{34}$ The first involves forcing the system at its resonance, which would be equivalent to exciting a jet close to the $\mathrm{K}-\mathrm{H}$ mode frequency/wave-number combination. In the second case, even for an off-resonance excitation, the underlying linear operator has a set of non-orthogonal eigenfunctions which, when considered in combination, can also produce a large response. This non-normality was discussed by McKeon and Sharma, ${ }^{19}$ in the case of turbulent pipe flows, where the role of critical layers was emphasized. Here, we discuss their observations by considering a 2D Cartesian $(x, y)$, incompressible, viscous and parallel flow, forced in the momentum equations, where normal modes allows the equations to be recast in the matrix form,

$$
\left(\begin{array}{c}
\tilde{u} \\
\tilde{v} \\
\tilde{p}
\end{array}\right)=\left(\begin{array}{ccc}
i \alpha(\bar{U}-c)-\frac{1}{R e} \nabla^{2} & \frac{\partial \bar{U}}{\partial y} & i \alpha \\
0 & i \alpha(\bar{U}-c)-\frac{1}{R e} \nabla^{2} & \frac{\partial \cdot}{\partial y} \\
i \alpha & \frac{\partial \cdot}{\partial y} & 0
\end{array}\right)^{-1} \quad\left(\begin{array}{c}
\tilde{f}_{x} \\
\tilde{f}_{y} \\
0
\end{array}\right),
$$

where $\nabla^{2}=-\alpha^{2}+\frac{\partial^{2}}{\partial y}$. For high Reynolds numbers $R e$, the linear operator becomes ill-conditioned at the critical layer, when the convective terms composing the main diagonal tend to zero. Additionally, the presence of $\frac{\partial \bar{U}}{\partial y}$ (in off-diagonal terms) clearly highlights that the operator is not self-adjoint. The shear, coupling the velocity components, is a source of non-normality that will become increasingly relevant close to the critical layer. Here, wave-like forces are considered, with the same phase speed and wave-number of the disturbances, illustrated in figure 5 . The responses will be explored numerically in the next sections.

\section{III.B. Direct forcing approach}

We consider the situation where the $\mathrm{K}-\mathrm{H}$ mode is stable, and we investigate the response of a stable mode to external force. The same 2D Cartesian $(x, y)$ system, used above to described the critical layer, is here considered. We fix a positive real value for the streamwise wave number $(\alpha=1.2)$ and vary the phase velocity $(c=0.1$ to 0.9$)$. The harmonic force is applied in different locations. The force is represented by a Dirac delta function ${ }^{\mathrm{e}} \delta\left(y-y_{f}\right)$. The direct problem can be expressed as

$$
\widetilde{\boldsymbol{q}}_{\alpha, \omega}=\mathcal{L}_{\overline{\boldsymbol{q}}, \alpha, \omega}^{-1} \widetilde{\boldsymbol{f}}_{\alpha, \omega}
$$

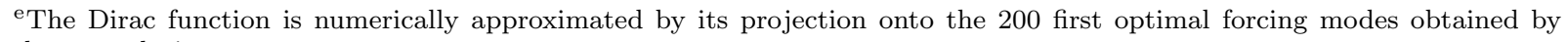
resolvent analysis. 


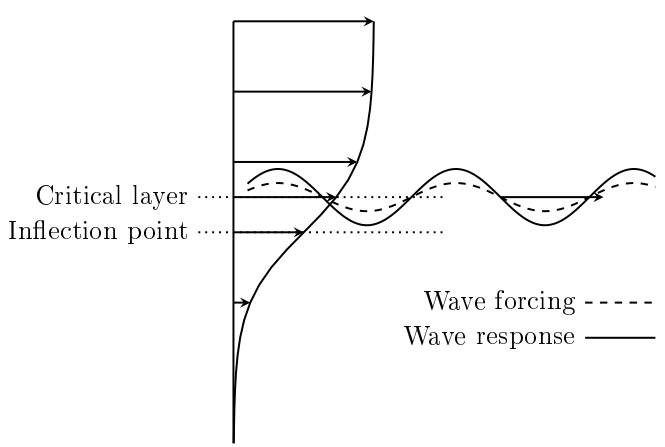

Figure 5. Scheme representing the ansatz of a wave travelling over the mean flow and of a forcing having the same wave structure in the streamwise direction than the perturbation. The critical layer is the position where the phase velocity coincides with the local mean velocity.

$N_{y}=200$ Chebyshev polynomials are used to approximate the differentiation matrix, and homogeneous boundary conditions are imposed in a stretched domain $y=[-H: H]$, with $H=5$. We first present the results considering a gain $G$, given by

$$
G=\frac{\left\|\widetilde{\boldsymbol{q}}_{\alpha, \omega}\right\|_{E}^{2}}{\left\|\widetilde{\boldsymbol{f}}_{\alpha, \omega}\right\|_{E}^{2}}
$$

with the energy norm defined as

$$
\left\|\widetilde{\boldsymbol{q}}_{\alpha, \omega}\right\|_{E}^{2}=\int_{-H}^{H}\left(\widetilde{u}^{*} \widetilde{u}+\widetilde{v}^{*} \widetilde{v}\right) \mathrm{d} y
$$

where $\cdot^{*}$ is the transpose-conjugate operation. $G$ is a measure of the maximum response of the linear system to external forcing localised in $y$ and periodic in $x$ and $t$. Results are considered for two different baseflows profiles (Couette and hyperbolic tangent). For each perturbation phase speed $c$, there is an associated critical-layer position, and all possible forcing positions $y_{f}$ are considered. Figure 6 shows maps of $G$. The position where force and critical layer coincide and the position of the inflection point, when it exists, are indicated. It is clear that, although the critical layer is recognised as a sensitive region, when this point coincides with an inflection point, the sensitivity is greater still.

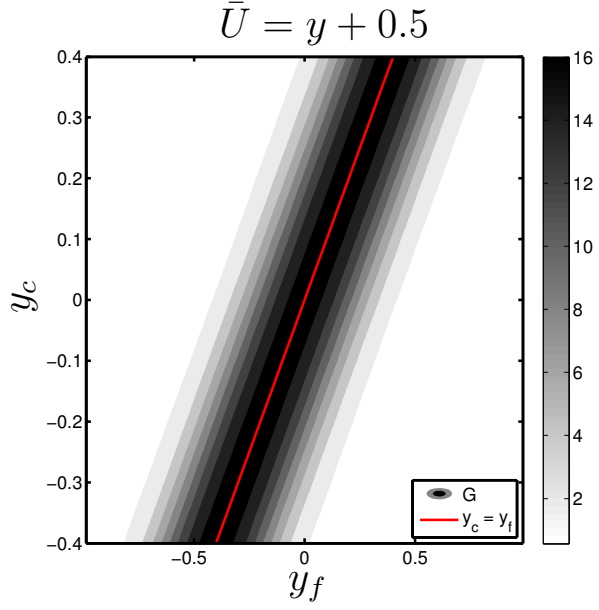

(a) Couette

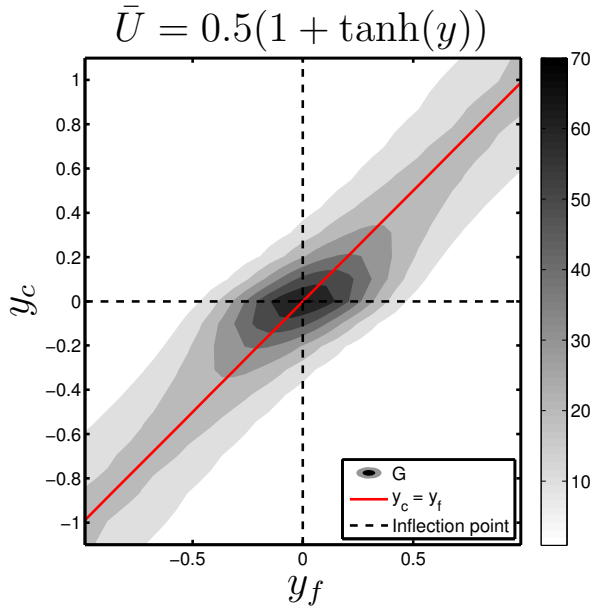

(b) Hyperbolic Tangent

Figure 6. Contour plots of $G$, when $y_{f}$ are swept, for all critical layer position associated to $c=0.1$ to $c=0.9$. Red solid line corresponds to the position where the force and the critical layer coincide. Dashed lines show the inflection point position when it exists.

Responses $\widetilde{u}$ are shown in figure 7 , for the Couette flow and in the hyperbolic tangent, when critical layer and inflection point are apart and coincide. The contours in both cases present the variation of the response with respect to the force position. The same behaviour discussed previously is observed: the 
maximum response happens at the critical layer and even more so if the critical-layer position coincides with the inflection point. A vortex-like structure and phase jump (not shown here) are observable. For example, a dip can be seen in figure 7(b) around the critical layer position for $y \sim-0.5$.

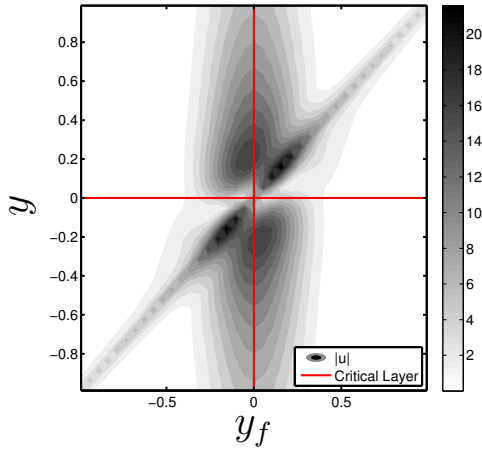

(a) Couette

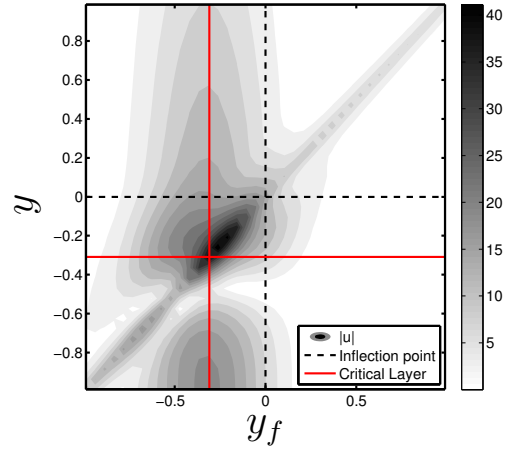

(b) Hyperbolic tangent for the critical layer which does not conincides with the inflection point

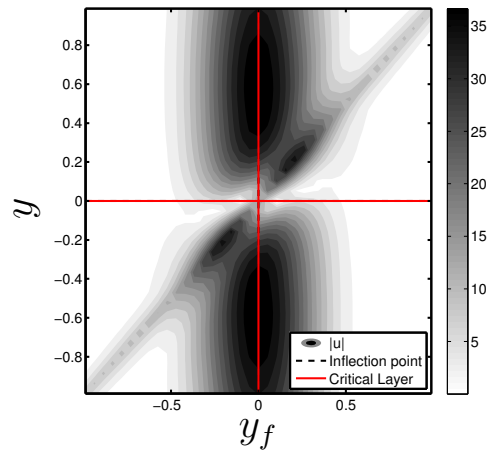

(c) Hyperbolic tangent for the critical layer which conincides with the inflection point

Figure 7. Response of the Dirac forcing $\delta\left(y-y_{f}\right)$. Critical layer away from the inflection point. Red solid line corresponds to the position where the force and the critical layer coincide. Dashed lines show the inflection point position when it exists.

\section{III.C. Resolvent analysis}

We now wish to consider more closely the forcing characteristics. The resolvent analysis provides the optimal forcing and response modes. This method is first presented in section III.C.1. In section III.C.2, we apply the resolvent analysis using the model problem presented in section III.B. Finally, this method is performed in a more realistic jet configuration in section III.C.3.

\section{III.C.1. Formulation of the resolvent analysis}

Let the output response $H \widetilde{\boldsymbol{q}}_{\alpha, \omega, m}$, where $\mathrm{H}$ is an observation operator, be rewritten in terms of the forcing as following

$$
H \widetilde{\boldsymbol{q}}_{\alpha, \omega, m}=H \mathcal{L}_{\overline{\boldsymbol{q}}, \alpha, \omega, m}^{-1} B \widetilde{\boldsymbol{f}}_{\overline{\boldsymbol{q}}, \alpha, \omega, m}=R_{\overline{\boldsymbol{q}}, \alpha, \omega, m} \widetilde{\boldsymbol{f}}_{\overline{\boldsymbol{q}}, \alpha, \omega, m},
$$

where $R_{\overline{\boldsymbol{q}}, \alpha, \omega, m}=H \mathcal{L}_{\overline{\boldsymbol{q}}, \alpha, \omega, m}^{-1} B$ is the resolvent operator. ${ }^{35}$ The goal of the analysis is to determine the forcing such that the norm of the associated response is maximised

$$
\max _{\widetilde{\boldsymbol{f}}_{\alpha, \omega, m}} \frac{\left\|H \widetilde{\boldsymbol{q}}_{\alpha, \omega, m}\right\|_{W}^{2}}{\left\|\widetilde{\boldsymbol{f}}_{\alpha, \omega, m}\right\|_{W}^{2}}=\frac{\left\|R_{\overline{\boldsymbol{q}}, \alpha, \omega, m} \widetilde{\boldsymbol{f}}_{\alpha, \omega, m}\right\|_{W}^{2}}{\left\|\widetilde{\boldsymbol{f}}_{\alpha, \omega, m}\right\|_{W}^{2}},
$$

where the weighted norm ${ }^{\mathrm{f}}\|\cdot\|_{W}$ is associated with the inner-product $(\cdot, W \cdot)$ with $W$ a definite positive Hermitian matrix, $(\cdot, \cdot)$ the Euclidean inner-product and $\|\cdot\|$ the Euclidean norm. The maximisation of the Rayleigh quotient (10) can be achieved by means of the following singular value decomposition

$$
W^{1 / 2} R_{\overline{\boldsymbol{q}}, \alpha, \omega, m} W^{-1 / 2}=U \Sigma V^{*},
$$

where $W^{1 / 2}$ is defined by the Cholesky decomposition $W=W^{1 / 2}\left(W^{1 / 2}\right)^{*}, U=\left(\boldsymbol{U}_{1}, \ldots, \boldsymbol{U}_{N}\right)$ and $V=$ $\left(\boldsymbol{V}_{1}, \ldots, \boldsymbol{V}_{N}\right)$ are orthonormal matrices and $\Sigma$ is a diagonal matrix. We define the optimal forcing modes $(\mathrm{OF})$ as $\boldsymbol{\Phi}_{i}=W^{-1 / 2} \boldsymbol{V}_{i}$ and the associated optimal response modes (OR) as $\boldsymbol{\Psi}_{i}=W^{-1 / 2} \boldsymbol{U}_{i}$. The Hankel singular values $\sigma_{i}$, diagonal elements of $\Sigma$ sorted in decreasing order, indicate the gains associated with forcing-response mode pairs. We obtain indeed the relationship

$$
H \mathcal{L}_{\overline{\boldsymbol{q}}, \alpha, \omega, m}^{-1} B \boldsymbol{\Phi}_{i}=\sigma_{i} \boldsymbol{\Psi}_{i}
$$

\footnotetext{
${ }^{\mathrm{f}}$ For simplicity, it is consider that the weighted norm is the same in the input and output space. Indeed, the forcing is a non-linear term and the output is the response. The full non-linear sketch figure 2 shows that they lie in the same space.
} 
which shows that the optimal response is that of the optimal forcing amplified by the corresponding Hankel singular value $\sigma_{i}$.

\section{III.C.2. Resolvent analysis in 2D incompressible shear layer}

Before presenting the results for the jet case, let us compare the solutions for the impulsive forcing with the resolvent of the 2D cases (Couette, hyperbolic tangent). For consistency, the previously defined gain $G$ equation (7) is also used as the resolvent norm. The $H$ and $B$ matrices here are given by,

$$
B=\left[\begin{array}{ll}
1 & 0 \\
0 & 1 \\
0 & 0
\end{array}\right], H=\left[\begin{array}{lll}
1 & 0 & 0 \\
0 & 1 & 0
\end{array}\right],
$$

such that forcing is restricted to the momentum equation, and the energy norm corresponds to the turbulent kinetic energy in an incompressible flow. Figure 8 presents the squared Hankel singular values (energy gain) $\sigma_{i}^{2}$, in logarithmic scale, from the resolvent of these two cases. This shows the relative importance of each mode pair, and the dominance of the first force/response mode of the orthogonal basis is clear.

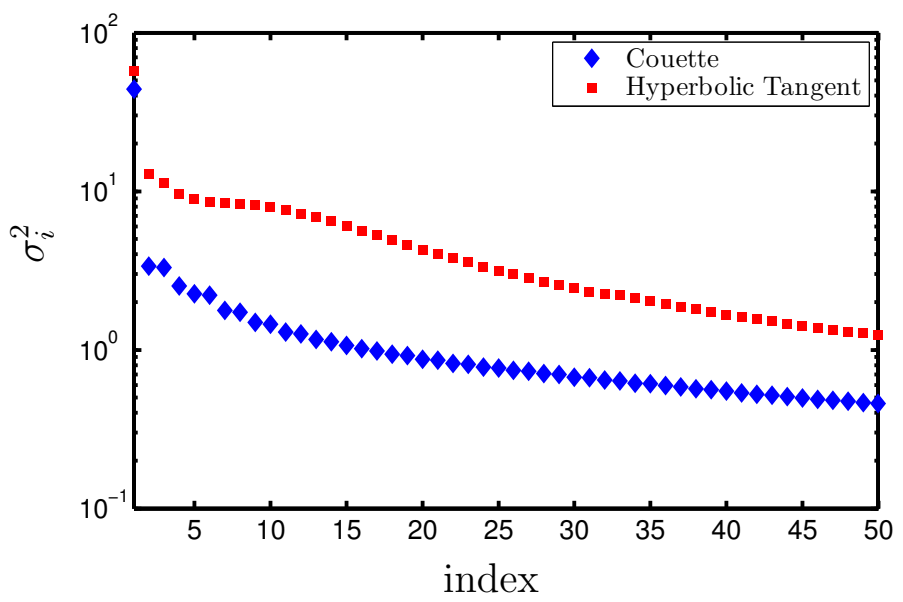

Figure 8. Hankel singular values of the resolvent analysis for the $2 \mathrm{D}$ Couette flow and the hyperbolic tangent.

The $\widetilde{u}$ velocity structure variation along the $y$ direction, from a Dirac forcing at the critical layer, is compared, in figure 9, with the optimal response given by the resolvent. A perfect match between both is not expected, but we note the main features obtained by the impulse response, i.e. the force at the critical layer generating the maximum response and the velocity dip present in the $\widetilde{u}$ velocity structure. This indicates that the optimal forcing/response mode pair is associated with a critical layer mechanism.

It can be noted that, even if the forcing responsible for the maximum response happens systematically at the critical layer, the dip in the response of the streamwise velocity $\widetilde{u}$ along the $y$ direction is found not to necessarily occur at the critical layer. The mismatch happens when an inflection point is present, but does not coincide with the critical layer.

\section{III.C.3. Resolvent analysis in an axisymmetric, compressible jet}

Resolvent analysis was shown in section III.C.2 to identify, in a locally parallel framework, the most amplified forcings and to highlight a critical-layer mechanism. Here, the method is applied in a locally parallel jet configuration and results compared with measurements. As discussed above, the linear wavepackets computed using the homogeneous linearised system closely match the measurement of a $M=0.4$ jet up to the end of the potential core, downstream of which the agreement worsens. The resolvent analysis is here used to explore if this discrepancy can be remedied by considering the optimally-forced wavepackets.

In performing the resolvent analysis, we assume the jet flow to be viscous, compressible, isothermal and axisymmetric in a locally parallel setting. The base flow comes from the measurement of a $M=0.4$ jet presented in Cavalieri et al. ${ }^{3}$ Due to the locally parallel flow assumption, only the axial component of the base-flow velocity is kept and the thermodynamic variables are determined using the Crocco-Busemann 


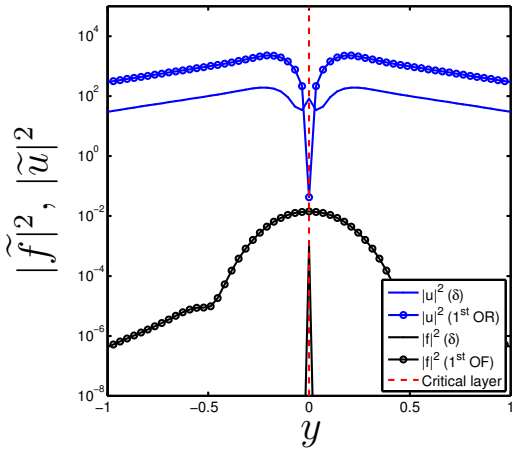

(a) Couette

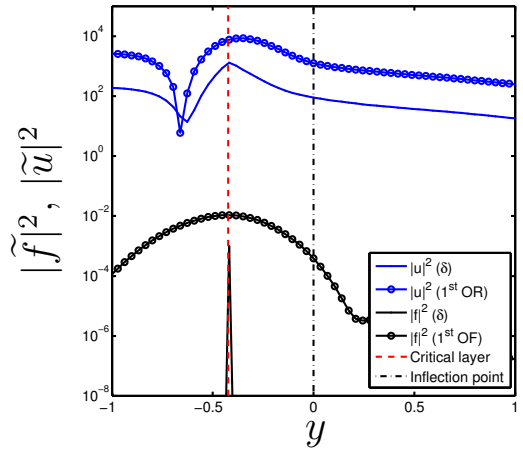

(b) Hyperbolic tangent for the critical layer which does not conincides with the inflection point

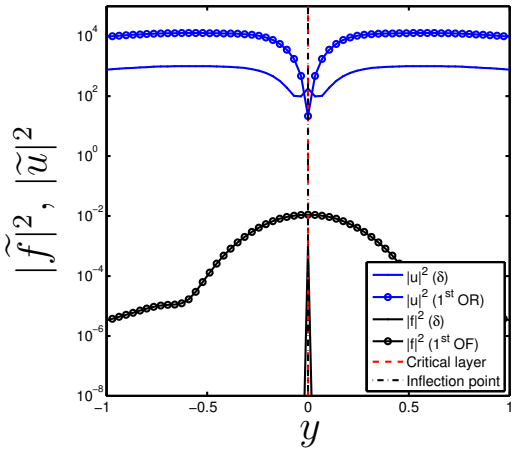

(c) Hyperbolic tangent for the critical layer which conincides with the inflection point

Figure 9. Comparison between a Dirac forcing at the critical layer and optimal forcing from resolvent analysis. Black lines correspond to the forcings and blue lines correspond to the responses. Solid lines are associated with the Dirac forcing and bullets are associated with the resolvent analysis. Vertical red dashed lines show the critical layer position and vertical black dashed black lines show the inflection point position when it exists.

relation and the perfect gas law, defining thus the base-flow as $\overline{\boldsymbol{q}}=(\bar{U}, 0, \bar{T}, \bar{\rho})^{T}$. The viscosity $\bar{\mu}$ is determined using the Sutherland's law. The effect of bulk viscosity is considered as $\mu_{B}=0.6 \mu$ for air in accordance with Freund. ${ }^{36}$ The equations are supplemented with a null Dirichlet boundary condition ${ }^{\mathrm{g}}$ in the farfield as was done in several previous works. ${ }^{39,40}$ The full set of equations in cylindrical coordinates under such assumptions is provided in appendix.

The equations are discretised and solved on a Chebyshev grid using a spectral collocation method ${ }^{41}$ in the radial direction. To compute the singular value decomposition of the resolvent operator, one should define the matrices $B$ and $H$, and the norm $\|\cdot\|_{W}$. To define the input-output matrices, we consider that the non-linearities act only on the momentum equation. Moreover, in order to simplify interpretation ${ }^{\mathrm{h}}$, we focus on outputs in the momentum equations also, which leads to the matrices,

$$
B=\left(\begin{array}{ll}
1 & 0 \\
0 & 1 \\
0 & 0 \\
0 & 0
\end{array}\right), \quad H=\left(\begin{array}{cccc}
1 & 0 & 0 & 0 \\
0 & 1 & 0 & 0
\end{array}\right)
$$

In compressible flows, as discussed in Hanifi et al. ${ }^{39}$ and Rowley et $a l_{.}^{42}$, there exists no unique choice for the norm definition. To obtain a meaningful energy norm, Hanifi et.al. suggested that the contribution of compression work to the total disturbance energy vanish since it is conservative. We follow this definition and define the energy norm as

$$
\|\widetilde{\boldsymbol{q}}\|_{E}^{2}=\int_{\Omega}\left(\frac{\bar{\rho}}{2}\left(\widetilde{u}^{*} \widetilde{u}+\widetilde{v}^{*} \widetilde{v}+\frac{\bar{\rho}}{2 \bar{T} \gamma} \widetilde{T}^{*} \widetilde{T}+\frac{\gamma-1}{2 \gamma} \frac{\bar{T}}{\bar{\rho}} \widetilde{\rho}^{*} \widetilde{\rho}\right) r \mathrm{~d} r=\widetilde{\boldsymbol{q}}^{*} W_{E} \widetilde{\boldsymbol{q}},\right.
$$

where $\gamma=1.4$ and the positive definite Hermitian matrix $W_{E}$ will be used to facilitate the computations in the $L_{2}$ norm. ${ }^{43} W$ is then defined as the upper diagonal block of $W_{E}$ such that $\left\|H^{*} H \widetilde{\boldsymbol{q}}\right\|_{E}^{2}=\|H \widetilde{\boldsymbol{q}}\|_{W}^{2}$.

In the following, we present the resolvent analysis, for a jet flow of $M=0.4$ and $R e=10000$. The phase velocity $c$ has been chosen as that predicted by a PSE computation for the same jet. The axisymmetric mode $m=0$ is considered, as it is the most acoustically effective, ${ }^{2,3}$ and $\mathrm{St}=0.6$ is arbitrarily chosen to illustrate the salient features of forced wavepacket behavior.

The resolvent analysis identifies a basis of forcing modes spanning $\operatorname{Ker}\left(H \mathcal{L}_{\overline{\boldsymbol{q}}, \alpha, \omega, m}^{-1} B\right)^{T}$ and a basis of response modes spanning $\operatorname{Im}\left(H \mathcal{L}_{\overline{\boldsymbol{q}}, \alpha, \omega, m}^{-1} B\right)$. Figure 10(a) shows the optimal energy gain $\sigma_{i}^{2}$ of the five

\footnotetext{
${ }^{\mathrm{g}}$ Applying a non-reflecting boundary condition is more accurate, using asymptotic ${ }^{37}$ or characteristic method. ${ }^{38}$ Nonetheless, the exponentially decaying modes in a large enough computation domain will generate only negligible reflected waves.

${ }^{\mathrm{h}}$ Numerical tests showed that the results are qualitatively insensitive if the output matrix $H$ is the identity matrix.
} 
first modes as a function of axial position. This figure shows that for all axial positions, the first optimal forcing/response mode pair clearly dominates the full response, similarly to what was observed for the model base-flows in section III.B. This suggests that forced wavepackets may be dominated by the optimal forcingresponse mode pair. Figure 10(b) shows the radial structure of the four first optimal forcing modes at $x / D=8$ and of the associated responses. Beyond the fact that the first optimal response mode dominates by one order of magnitude, the shape of the forcing and the associated response are typical of the critical layer mechanism highlighted section III.B. Indeed, even if the forcing shows a broad shape, its maximum is at the critical layer and the response has one dip and a phase jump (phases are shown in appendix, figure 21 ), sign of a vortex-like structure. The higher modes show several dips and can be interpreted as functions which span the response to less relevant turbulent structures. In what follows we thus consider only the first resolvant mode. The dominance of the first mode is encouraging vis-à-vis an eventual reduced-order model.

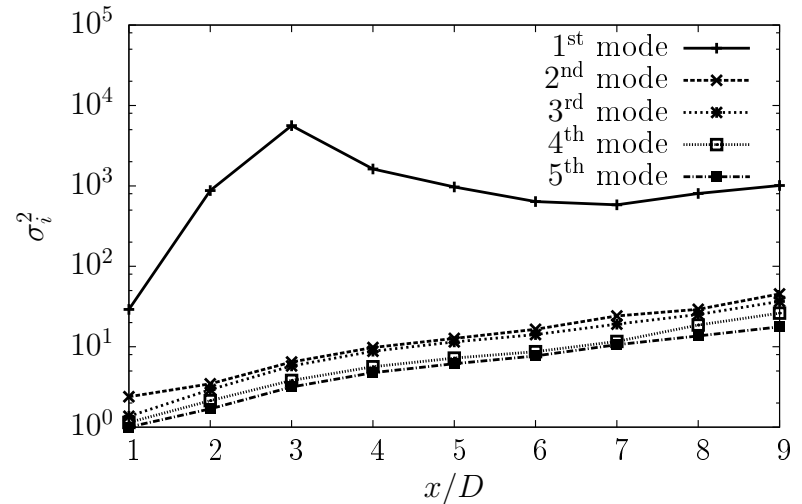

(a) Optimal response energy gain $\sigma_{i}^{2}$ for all axial positions for St $=0.6$.

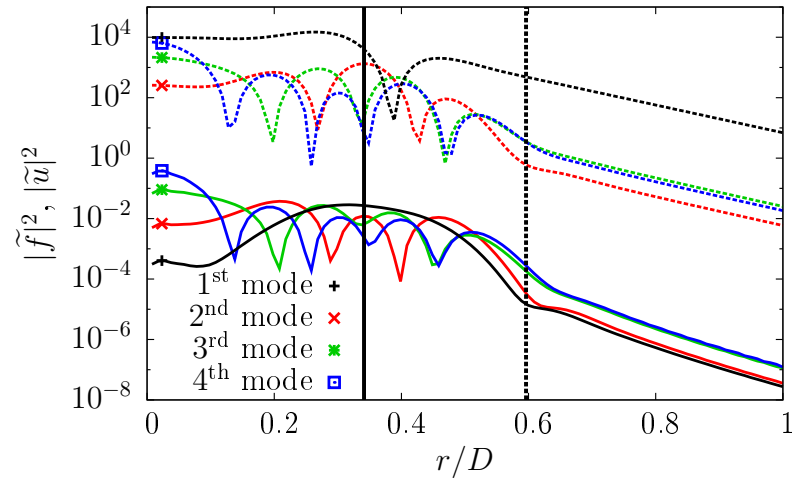

(b) Optimal forcing/response at $x / D=8$. For lisibility, the forcing modes $\boldsymbol{\Phi}_{i}$, in solid lines, has been multiplied by $10^{-3}$. The associated responses $\sigma_{i} \boldsymbol{\Psi}_{i}$ are in dashed lines. Vertical solid: critical layer, vertical dashed: inflection point.

Figure 10. Characterisation of the optimal forcing/response modes.

In figure (11), we show the power spectral density (PSD) of the axial perturbation velocity obtained by the resolvent analysis compared to time-resolved PIV measurements (TRPIV), solutions of the linearised Euler equations (LEE) from Zhang et al. ${ }^{4}$ and PSE computation at two emblematic positions: $x / D=3$, which typifies results upstream of end of the potential core, where the homogeneous linear framework matches data nicely, and $x / D=8$, typifying results obtained downstream of the potential core where linear theory fails. All positions are shown in appendix figure 20. In the experimental results, upstream of $x / D \sim 5$, the first POD mode of the TRPIV data for $m=0$ shows that the turbulent jet flow comprises a vortex-like signature in this region. ${ }^{2}$ We can also see that, in this region, the linear wavepacket models (PSE and LEE) correctly capture this structure, as these curves collapse with the TRPIV data. This means that the homogeneous linear wavepacket model is able to predict the near-nozzle behaviour of the K-H instability, which presents exponential growth in $x$. Moreover, the resolvent optimal response modes, though corresponding to a forcedwavepacket scenario, clearly dynamically different from the homogeneous linear wavepackets, also match the measurement upstream of $x / D \sim 5$. Here, $\mathcal{L}_{\overline{\boldsymbol{q}}, \alpha, \omega, m} \widetilde{\boldsymbol{q}}_{\omega, m}=0$ is already respected, and the optimal forcing is also itself approximately a solution of the homogeneous equation. Non-linearities within the turbulent flow also act in this region predominantly by forcing the Kelvin-Helmholtz mode.

However, downstream of the potential core, the homogeneous linear models (PSE or LEE) predict a quitedifferent radial structure, as can be seen at $x / D=8$ figure 11 or in (e)-(h) of figure 20 . The measurement shows that the flow field in these regions retains a vortex-like structure possessing a local dip around $r / D \sim$ 0.4 (the dip corresponds to the phase jump of $u$ in the radial direction) which, is not captured by the linear homogeneous wavepacket model. With a non-linear forcing term allowed, the resolvent mode has an optimal response whose radial structure is similar to that of the experimentally educed wavepacket. This is an encouraging indication that the notion of forced linear wavepackets may correspond to what is happening in the flow downstream of the potential core. We note here that the position of the dip doesn't always correspond exactly with experimental results. This position is highly sensitive to the perturbation phase velocity $c$, which fixes the critical layer position, where the optimal forcing is systematically maximum. Note that the phase 
velocity chosen for resolvent analysis comes from linear PSE, which does not match experiments in the downstream region. The forced wavepacket, which we assume to be present in experiments, can have a phase velocity slightly different from the unforced wavepacket, explaining the observed discrepancies. This point has to be verified in further studies.
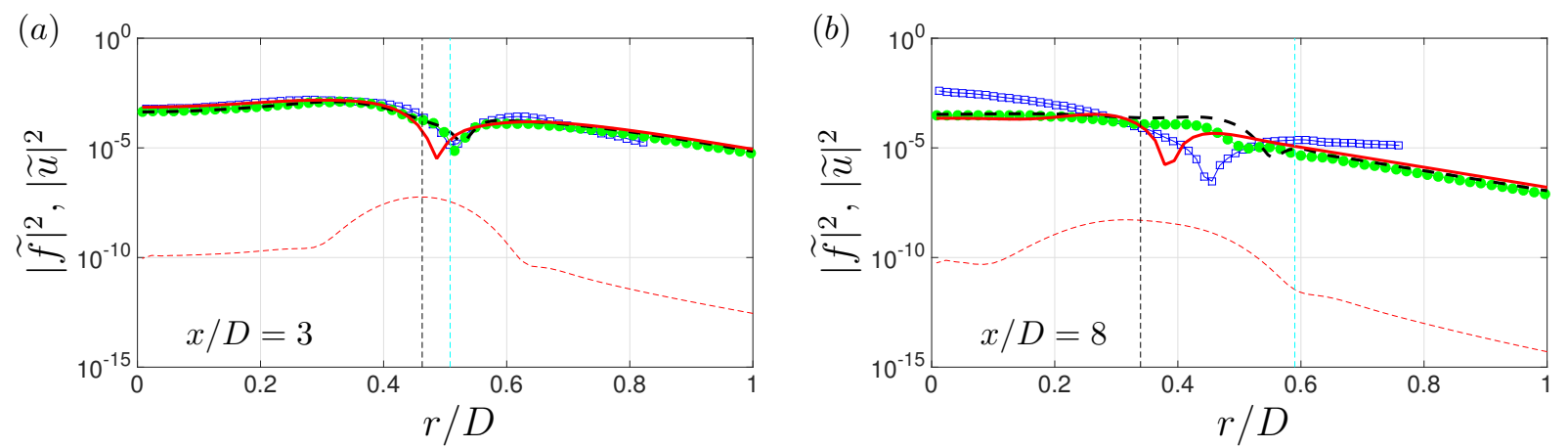

Figure 11. Comparison of optimal forcing modes (red dashed lines) and optimal response modes (red solid lines) of axial velocity fluctuation at $x / D=3$ and $x / D=8$ with experimental data (blue squares) and the solution of the homogeneous linear problem (LEE: green line with solid dots ; PSE: black dashed line). St $=0.6, R e=10000, M=0.4$. The phase velocity $c$ predicted by PSE is used. The vertical dashed black line is the critical layer position and the vertical dashed cyan line the inflectional point position.

The above results can be presented in a more compact manner. We can compare TRPIV, PSE and OR of RA by defining a metric $\beta$, (taken here between PSE and RA for example)

$$
\beta_{P S E-R A}(x, \mathrm{St})=\frac{\left(H \widetilde{\boldsymbol{q}}_{P S E}, \boldsymbol{\Psi}_{1}\right)_{r}}{\sqrt{\left(H \widetilde{\boldsymbol{q}}_{P S E}, H \widetilde{\boldsymbol{q}}_{P S E}\right)_{r}\left(\mathbf{\Psi}_{1}, \mathbf{\Psi}_{1}\right)_{r}}},
$$

where

$$
(\boldsymbol{a}, \boldsymbol{b})_{r}=\int_{r_{b}}^{r_{t}} a^{*}(r) b(r) r \mathrm{~d} r
$$

where $r_{b}$ and $r_{t}$ are the integration range for each variable. For the axisymmetric mode considered here, $u$ and $v$ are involved in the calculation. The results of the absolute values of $\beta(|\beta|)$ between TRPIV and PSE, TRPIV and OR and PSE and OR are shown figure 12. When two quantities have similar radial structure, the value of $|\beta|$ is high, and vice-versa.

In the panel (a) of figure (12), we see that PSE matches the experimental data well before the end of the potential core, especially for $\mathrm{St}=0.3 \sim 0.9$. This result has been noted in Cavalieri et al. ${ }^{3}$ for example. For the correlation between TRPIV and OR, we see in panel (b) that even though OR is not matching the measurement as well as PSE before the potential core (due to the aforesaid sensitivity to the precise value of the phase velocity used to compute OR), downstream of the potential core, the agreement of OR with TRPIV is higher than that of PSE. This result has already been inferred and discussed from the figure 11 above, but figure 12 is a useful means by which to compact the comparisons for all frequencies and axial positions into a single graphic. Finally, $|\beta|$ between PSE and RA is shown in panel (c), from which we can see that the correlation between these two models is good before the end of the potential core for all St, except around $x / D=1$, where the agreement is worse.

Figure 13(a) shows a map ${ }^{i}$ of the optimal forcing mode $\boldsymbol{\Phi}_{1}$, whose norm is fixed to one. The optimal forcing diligently follows the critical layer. The associated response $\sigma_{1} \boldsymbol{\Psi}_{1}$ is shown figure 13(b). The response also follows the critical layer and we note that in general the dip is found to be located between the critical layer and the inflection point. A maximum response appears at $x / D=3$, which is consistent with the peak at the same position of the optimal energy gain figure 10(a).

To summarise, the first optimal forcing mode dominates the higher order ones and shows a maximum at the critical layer for all axial positions. The response seems to be consistent with the flow behavior downstream of the potential core, supporting the idea that a forced wavepacket model may be what is needed to describe features present in the jet downstream of the potential core. Finally a spot of high

\footnotetext{
${ }^{\mathrm{i}}$ We have to keep in mind that this map comes from study performed in a locally parallel flow assumption. It shouldn't be interpreted as figure 16 taking into account the convective behavior of the flow.
} 

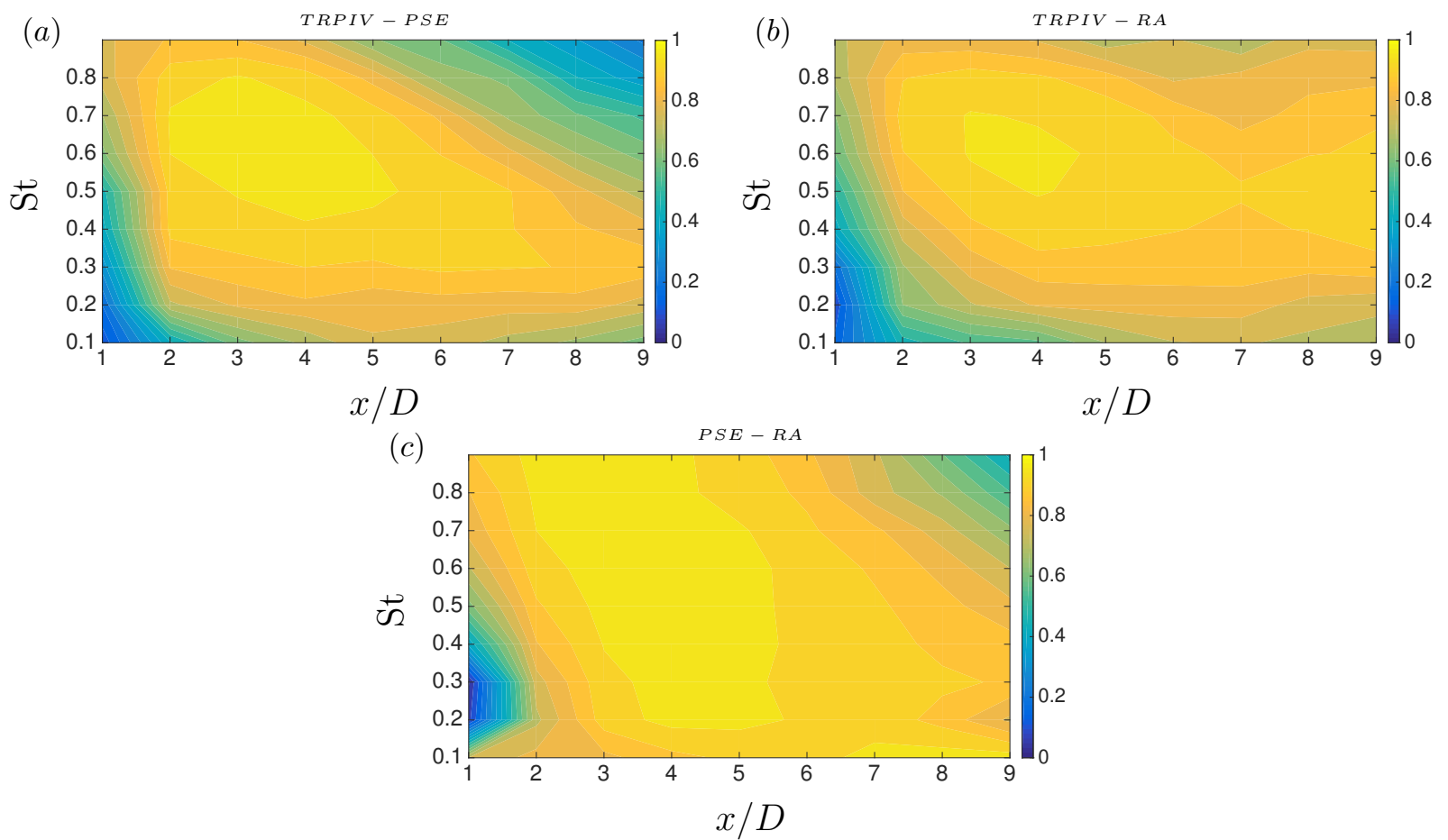

Figure 12. Values of $|\beta|$ at $R e=10000, M=0.4, m=0 . c$ is determined from the PSE calculation.

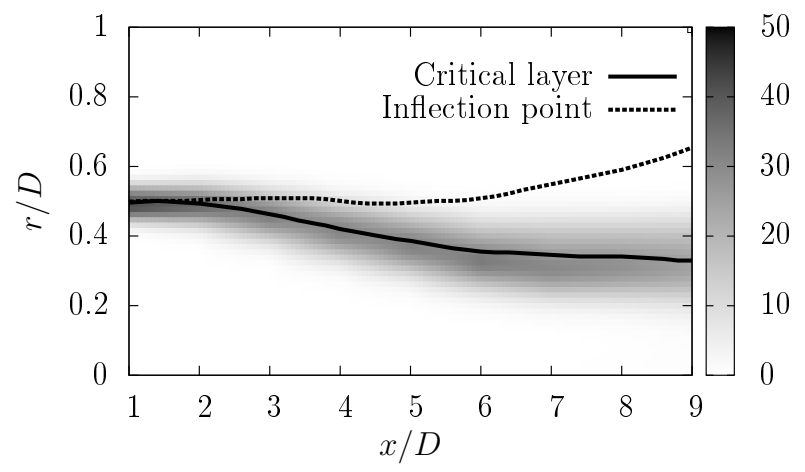

(a) Optimal forcing mode $\boldsymbol{\Phi}_{1}$.

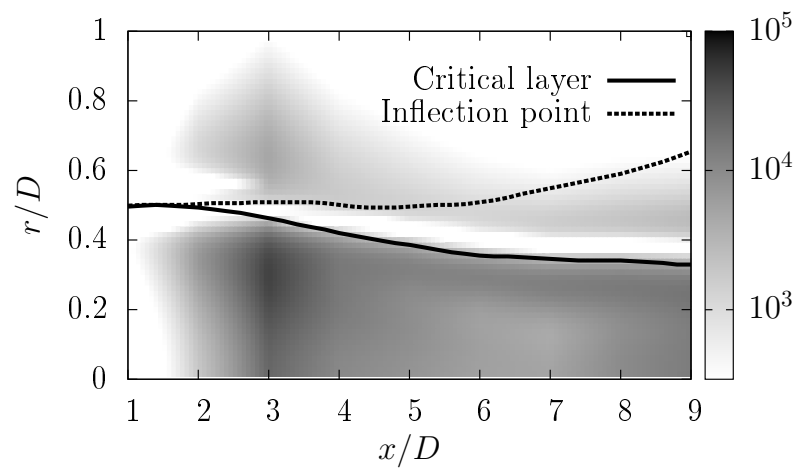

(b) Optimal repsonse mode $\sigma_{1} \boldsymbol{\Psi}_{1}$.

Figure 13. Power spectral density of the first optimal forcing and response modes along axial direction at St $=0.6, c$ determined by PSE. Solid line is the critical layer position and dashed line is the inflection point position. 
sensitivity to forcing is found upstream the potential core at $x / D=3$ for $\mathrm{St}=0.6$. Because of the locally parallel assumption of the resolvent analysis, conclusions about relationships between different axial positions due to the convective nature of the flow can be hard to draw. In section III.D, we will complement the study with a more global approach, the PSE-4D-Var.

\section{III.D. PSE-4D-Var}

In this section we drop the parallel-flow assumption and consider a slowly diverging jet using the parabolised stability equations. We here explore the missing pieces of the linear model using a data-driven approach, 4DVar. We use a specific variant of this, called weak constraint variational data assimilation. ${ }^{44}$ This estimation problem is formulated as an optimisation problem, where we authorise ${ }^{j}$ modifications of an "external" forcing considered as modelling uncertainties. The "external" forcing can be interpreted as a non-linear term, not taken into account by the linear model, and which we would like to identify. Thus, we are searching, via an inverse problem, the minimum forcing, interpreted as the effect of non-linearities, necessary to match measurements. In what follows, for the sake of simplicity, we do not solve the entire minimisation problem, but restrict ourselves to determining the sensitivity of the linear PSE solution with respect to an external forcing introduced in order to coax that solution towards the experimental data.

\section{III.D.1. Problem formulation}

The parabolised stability equations ${ }^{45}$ allow instability wave evolution over a slowly varying base flow to be modeled. The formulation used here is similar to that used in previous work. ${ }^{3,28,37}$ The assumption of a slowly varying base-flow allows ${ }^{46}$ us to decompose the perturbation associated with the mode $(\omega, m)$ into slowly and rapidly varying (wavelike) parts, ${ }^{45}$

$$
\widetilde{\boldsymbol{q}}_{\omega, m}(x, r)=\boldsymbol{q}(x, r) e^{i \int_{0}^{x} \alpha(\xi) \mathrm{d} \xi} .
$$

$\boldsymbol{q}(x, r)$ is the slowly varying part and $e^{i \int_{0}^{x} \alpha(\xi) \mathrm{d} \xi}$ is the wavelike part. The $(\omega, m)$ index is dropped for compactness. We introduce the decomposition (18) into the compressible Navier-Stokes equations. The non-linear terms are then neglected assuming small perturbations, and the first axial derivatives of $\alpha$ and the second axial derivatives of $\boldsymbol{q}$ are neglected assuming a slow variation of these variables. We obtain thus an equation of the form

$$
E \frac{\partial \boldsymbol{q}}{\partial x}+(A+\alpha B) \boldsymbol{q}=0,
$$

where the matrix $A$ contains discrete radial derivatives. The domain $x / D=[0, L], r / D=[0, \infty]$ is discretised using $N_{x}$ points in the axial direction and $N_{r}$ Chebyshev collocation points in the radial direction. Details of derivation of the PSE for compressible flow in cylindrical coordinates are given in Sasaki. ${ }^{28}$ Decomposition (18) is a priori not unique because of the presence of the $x$ variable in $\boldsymbol{q}(x, r)$ as well in $\alpha(x)$ and because no evolution equation is given for $\alpha$. In order to overcome this ambiguity, a normalisation constraint is added (see Herbert ${ }^{45}$ ) such that the exponential dependence (real and imaginary) is absorbed by the wavelike term $e^{i \int_{0}^{x} \alpha(\xi) \mathrm{d} \xi}$ :

$$
\left(\boldsymbol{q}, \frac{\partial \boldsymbol{q}}{\partial x}\right)_{r}=\int_{0}^{\infty} \boldsymbol{q} \cdot \frac{\partial \boldsymbol{q}}{\partial x} r \mathrm{~d} r=0 .
$$

Starting with $\left(\boldsymbol{q}_{0}, \alpha_{0}\right)$, often taken as the Kelvin-Helmoltz mode, solution of the linear stability equations, $\boldsymbol{q}(x, r)$, can be obtained by integrating equation (19) in space using an implicit Euler scheme, with iterations in $\alpha$ such that the constraint (20) is respected. ${ }^{37}$ The linear PSE procedure allows wavepackets for each frequency-azimuthal mode pair to be propagated independently.

In the 4D-Var under weak dynamical constraint, we would like the solution, $\boldsymbol{q}(x, r)$, to follow the observations, and we consider that this solution respects the model - here the linear PSE - only in a weak sense. In order to relax this constraint, we introduce a forcing term $\boldsymbol{f}$, interpreted as the effect of non linearities, as discussed in section II. Combining the nonlinear forcing with equations (19) and (20), the forced parabolised

jUsually, we also authorize modifications of the initial condition because it is a sensitive parameter, but this ability of the method is not exploited in this paper. 
stability equations can be written as

$$
\left\{\begin{array}{l}
E \frac{\partial \boldsymbol{q}}{\partial x}+(A+\alpha B) \boldsymbol{q}=\boldsymbol{f} \\
\left(\boldsymbol{q}, \frac{\partial \boldsymbol{q}}{\partial x}\right)_{r}=\boldsymbol{q}^{*} W_{r} \frac{\partial \boldsymbol{q}}{\partial x}=0
\end{array}\right.
$$

where $W_{r}$ is the discrete weight matrix associated with the inner-product $(\cdot, \cdot)_{r}$. Note here that we implicitly enforce the shape of the forcing of the wavepacket as

$$
\widetilde{\boldsymbol{f}}_{\omega, m}(x, r)=\boldsymbol{f}(x, r) e^{i \int_{0}^{x} \alpha(\xi) \mathrm{d} \xi},
$$

and we can define a forcing in physical space as

$$
\boldsymbol{f}_{\phi}(x, r, \theta, t)=\sum_{\omega} \sum_{m} \tilde{\boldsymbol{f}}_{\omega, m}(x, r) e^{i(m \theta-\omega t)} .
$$

This means that because a harmonic framework is used, the optimal forcing is constrained to have a wavepacket shape. This shape is indeed able to act efficiently on the instability wave because a forcing whose wavenumber matches that of the wavepacket should lead to the most significant dynamic effects. In principle, $f$ could come, for instance, from a non-linear PSE computation taking account of a reduced number of wave-wave interactions. In the present study all possible non-linear interactions are potentially considered via the inverse problem.

The objective of $4 \mathrm{D}$-Var is to seek $\boldsymbol{f}$ minimising

$$
\mathcal{J}(\boldsymbol{q}, \alpha, \boldsymbol{f})=\frac{1}{2} \int_{0}^{L}\|\mathbb{H}(\boldsymbol{q}, \alpha)-\mathcal{Y}\|_{W_{o}}^{2} \mathrm{~d} x+\frac{1}{2}\left\|\mathbb{H}\left(\boldsymbol{q}_{L}, \alpha_{L}\right)-\mathcal{Y}_{L}\right\|_{W_{T}}^{2}+\frac{1}{2} \int_{0}^{L}\|\boldsymbol{f}\|_{W_{f}}^{2} \mathrm{~d} x,
$$

where $\mathbb{H}(\boldsymbol{q}, \alpha)$ is an observation operator ${ }^{\mathrm{k}}$ allowing passage from the state space to the observation space. $\mathcal{Y}$ are associated observations, i.e. experimental measurements. We choose here:

$$
\mathbb{H}(\boldsymbol{q}, \alpha)=Q\left|\boldsymbol{q} e^{i \int_{0}^{x} \alpha(\xi) \mathrm{d} \xi}\right|^{2},
$$

where $Q$ is a rectangular matrix of zeros and ones. Similarly to the output matrix $H$, equation (14), each line of $Q$ contains a "one" which selects the components and the radial positions which are observed, here the axial and radial velocity for the positions where there are available TRPIV results. $\boldsymbol{q}_{L}, \alpha_{L}$ and $\mathcal{Y}_{L}$ refer to the values of the variables or parameters at $x / D=L$. Indeed, we penalise the terminal condition in order to avoid transient behavior at the end of the domain of the adjoint equation, presented later. The norms $\|\cdot\|_{W_{o}},\|\cdot\|_{W_{T}}$ and $\|\cdot\|_{W_{f}}$ are respectively associated with the inner-products $\left(Q^{*} \cdot Q^{*} W_{o} \cdot\right)_{r},\left(Q^{*} \cdot, Q^{*} W_{T} \cdot\right)_{r}$ and $\left(\cdot, W_{f} \cdot\right)_{r}$. The weight matrices $W_{o}, W_{T}$ and $W_{f}$ allow different degrees of confidence to be specified in the observations or in the PSE model. Finally, we define, for any $M \in \mathbb{R}^{N_{c} N_{r} \times N_{c} N_{r}}$, the adjoint matrix $M^{+}=W_{r}^{-1} M^{*} W_{r}$, such that $(M \boldsymbol{x}, \boldsymbol{y})_{r}=\left(\boldsymbol{x}, M^{+} \boldsymbol{y}\right)_{r}$.

\section{III.D.2. Optimality system}

The minimisation problem exposed in section III.D.1 is a constrained optimisation problem. It consists in minimising (24) under the constraint (21). This problem is usually solved using a quasi-Newton algorithm, which requires the gradient of the cost functional $\mathcal{J}$ with respect to the control parameter $f$. Because the control parameter space is of large dimension, we use an adjoint method, where the gradient of the cost functional $\mathcal{J}$ is determined by the integration of an adjoint equation. ${ }^{47}$ The direct and adjoint systems are then solved iteratively until convergence to the optimal solution.

The adjoint PSE can be determined in different ways. The first consists in "parabolising" the adjoint linearised Euler equations as done by Dobrinsky ${ }^{48}$ for a receptivity problem. The second is to directly derive the adjoint PSE as in Pralits et al. ${ }^{49}$ for a sensitivity analysis or as in Pralits et al..$^{50}$ and Airiau et al. ${ }^{51}$ for flow control. For reasons of consistency between PSE and adjoint PSE, the latter method has been chosen.

\footnotetext{
${ }^{\mathrm{k}}$ The observation operator $\mathbb{H}(\boldsymbol{q}, \alpha)$ is related to the output matrix $H$, introduced section II because both define the output space. Here, it is simply generalized to a non-linear operator.
} 
In order to derive the adjoint equation, we introduce additional variables $\boldsymbol{\lambda}$ and $\zeta$, called Lagrange multipliers, whose role is to enforce the constraint (21). We then transform the constrained optimisation problem to an unconstrained optimisation problem, defining the Lagrangian

$$
\begin{aligned}
\mathcal{L}(\boldsymbol{q}, \alpha, \boldsymbol{f}, \boldsymbol{\lambda}, \zeta)= & \mathcal{J}(\boldsymbol{q}, \alpha, \boldsymbol{f}) \\
& -\operatorname{Real}\left(\int_{0}^{L}\left(E \frac{\partial \boldsymbol{q}}{\partial x}+(A+\alpha B) \boldsymbol{q}-\boldsymbol{f}, \boldsymbol{\lambda}\right)_{r} \mathrm{~d} x\right) \\
& -\operatorname{Real}\left(\int_{0}^{L}\left(\boldsymbol{q}, \frac{\partial \boldsymbol{q}}{\partial x}\right)_{r} \zeta \mathrm{d} x\right) .
\end{aligned}
$$

The derivation of the Lagrangian with respect to each variable is detailed in the appendix and leads to the following optimality system.

DIRECT SYSTEM:

$$
\left\{\begin{array}{l}
E \frac{\partial \boldsymbol{q}}{\partial x}+(A+\alpha B) \boldsymbol{q}=\boldsymbol{f} \\
\left(\boldsymbol{q}, \frac{\partial \boldsymbol{q}}{\partial x}\right)_{r}=0 .
\end{array}\right.
$$

ADJOINT SYSTEM:

$$
\left\{\begin{array}{l}
-E^{+} \frac{\partial \boldsymbol{\lambda}}{\partial x}+\left(A+\alpha B-\frac{\partial E}{\partial x}\right)^{+} \boldsymbol{\lambda}+\frac{\partial \boldsymbol{q}}{\partial x}\left(\zeta-\zeta^{*}\right)-\boldsymbol{q} \frac{\partial \zeta^{*}}{\partial x}=R H S_{1}, \\
(B \boldsymbol{q}, \boldsymbol{\lambda})_{r}=R H S_{2}, \\
E^{+} \boldsymbol{\lambda}(L, r)=R H S_{3}, \\
\zeta(L)=0 .
\end{array}\right.
$$

$R H S_{1}, R H S_{2}$ and $R H S_{3}$ are expressed in the appendix and depend on the definition of the cost functional (24).

\section{OptimaLity CONDITION:}

$$
\frac{\partial J}{\partial \boldsymbol{f}}=\boldsymbol{\lambda}(x, r)+W_{f} \boldsymbol{f}
$$

The minimisation problem can be solved iteratively ${ }^{52}$ in a computationally efficient manner using this optimality system. Once the direct system $(29)$ is solved, $(\boldsymbol{q}, \alpha)$ are known and one can integrate the adjoint system (30) backwards in space to obtain $(\boldsymbol{\lambda}, \zeta)$, and finally obtain the gradient $\frac{\partial J}{\partial f}$ using the optimality condition (31). Using a quasi-Newton algorithm, $\boldsymbol{f}$ can be updated and a new iteration can begin. The procedure stops when convergence is reached, giving access to the optimal forcing $\boldsymbol{f}$.

We would like to avoid issues of interpretation associated with the numerical minimisation procedure and the definition of the cost functional, especially due to the choice of the penalty terms. That is why, in the scope of this paper, we restrict the study to a sensitivity analysis. We explore the effect of a small forcing of the PSE and which improves the model with respect to the observations. However, the gradient of the cost functional $\mathcal{J}$ with respect to the wavepacket forcing $\widetilde{\boldsymbol{f}}_{\omega, m}$ can be ambiguous to define and to interpret because of the scale separation assumption (18). We choose therefore to define the forcing which will be used at the second iteration step of the optimisation problem. Considering a Newton-like gradient algorithm, the $(n+1)^{\text {th }}$ forcing term is found using the following rule

$$
\boldsymbol{f}^{(n+1)}=\boldsymbol{f}^{(n)}-\gamma^{(n)}\left(\frac{\partial J}{\partial \boldsymbol{f}}\right)^{(n)},
$$

where the relaxation parameter $\gamma^{(n)}$ is found using a line-search algorithm. ${ }^{53}$ Because we consider the first iteration, $\boldsymbol{f}^{(1)}=0$, and a step sufficiently small such that $\alpha$ varies sufficiently slightly, we have the forcing in physical space expressed as

$$
\widetilde{\boldsymbol{f}}_{\omega, m}^{(2)} \approx-\gamma^{(1)}\left(\frac{\partial J}{\partial \boldsymbol{f}}\right)^{(1)} e^{i \int_{0}^{x} \alpha^{(1)}(\xi) \mathrm{d} \xi}
$$


We define then the following variable

$$
\delta \tilde{\boldsymbol{f}}_{\omega, m}=-\left(\frac{\partial J}{\partial \boldsymbol{f}}\right)^{(1)} e^{i \int_{0}^{x} \alpha^{(1)}(\xi) \mathrm{d} \xi},
$$

which represents the direction of the small forcing of the linear PSE, expressed in physical space, and which will improve the cost functional. This infinitesimal forcing, defined up to a constant, will be referred to henceforth as the forcing sensitivity of the cost functional $\mathcal{J}$. An infinitesimal response $\delta \widetilde{\boldsymbol{q}}_{\omega, m}$ can be defined as well. Let $\widetilde{\boldsymbol{q}}_{\omega, m}^{h}$ be the solution of the homogeneous PSE and $\widetilde{\boldsymbol{q}}_{\omega, m}^{f}$ the solution of the PSE forced by $\gamma \delta \widetilde{\boldsymbol{f}}_{\omega, m}$ with $\gamma=10^{-8}$, we define $\delta \widetilde{\boldsymbol{q}}_{\omega, m}=\widetilde{\boldsymbol{q}}_{\omega, m}^{f}-\widetilde{\boldsymbol{q}}_{\omega, m}^{h}$.

\section{III.D.3. Results}

Sensitivity of the error of PSE with respect to an external forcing is here explored for the $M=0.4$ round jet presented in Cavalieri et al. ${ }^{3}$ the same used for the resolvent analysis in section III.C. The axisymmetric wavepacket $(m=0)$ at $\mathrm{St}=0.6$ is used in this section as an example to explore salient traits of the problem. The observations considered are power spectral density of the axial and radial components of the TRPIV measurements radially filtered by POD. ${ }^{3}$ The observation of the axial component at the centerline, $\widetilde{u}_{r=0}$, is compared in figure 14(a) with the values predicted by PSE. It shows the good agreement of the growth of the instability wave, until this wave becomes neutrally stable at around $x / D \sim 5$ and then begins to decay, while experiments show an increase after the end of the potential core. Figure 14(b) shows the real and imaginary part of the local wavenumber $\alpha$ predicted by PSE.

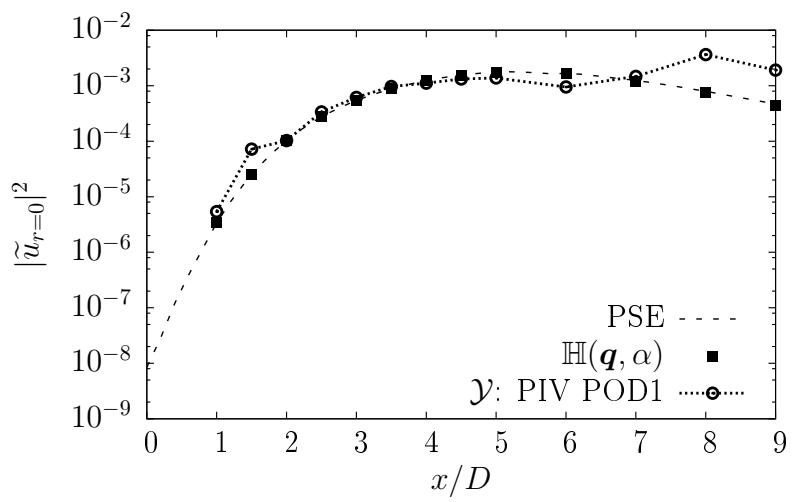

(a) Centerline axial fluctuating kinetic energy. Comparision between the observations $\mathcal{Y}$ (line with circles) with the PSE (dashed line) and the associated observation operator $\mathbb{H}(\boldsymbol{q}, \alpha)$ (squares).

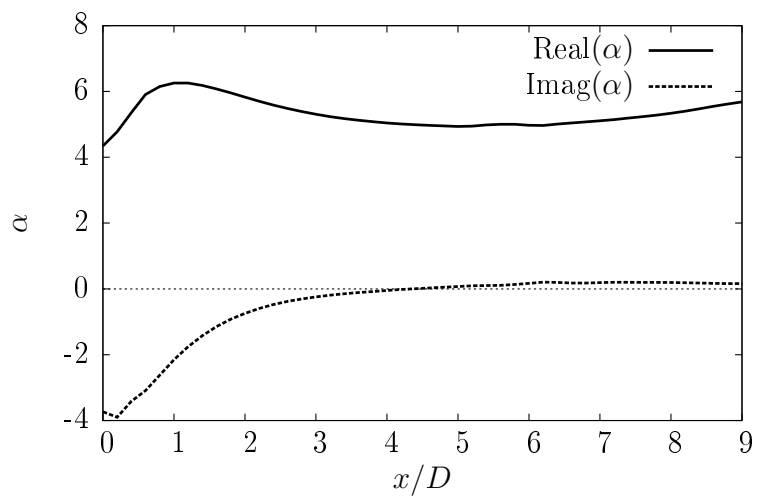

(b) Local wavenumber predicted by PSE. Real part and imaginary part.

Figure 14. Characterisation of the observations and of the PSE results.

Figures 15(a) and 15(b) show $x-r$ maps of the observed (experimental) power spectral densities of the axial and radial components respectively, while figures $15(\mathrm{c})$ and $15(\mathrm{~d})$ are axial and radial components of PSE results. These plots show again how linear PSE and experiments match until approximately $x / D \sim 4$. Then, looking at the PSD of the axial velocities of the observations (figure 15(a)) and PSE (figure 15(c)), we note that PSE has higher values around $(x / D=5, r / D=0.25)$ than the experiments. The experiments, on the other hand, have a big increase of axial fluctuating kinetic energy around the centerline at $x / D=8$, while PSE decays. These differences are clearly visible in the error map figure 16(a). Moreover, this error map shows that, downstream of the potential core, an error follows the critical layer until the end of the domain.

Superimposed on the error in figure 16(a) is the forcing $\left|\delta \widetilde{\boldsymbol{f}}_{\omega, m}\right|^{2}$, the position of the critical layer (where $\operatorname{Real}(c)=U$ ) and the position of the inflection point of the mean profile. Associated with these errors, two spots of high sensitivity to forcing can be observed. Both are upstream of locations of disagreement between linear PSE and experiments. The first, denoted $\mathcal{S}_{1}$, peaks around $(x / D=3, r / D=0.45)$, comprises a wide spatial shape and seems to follow the critical layer. The second, denoted $\mathcal{S}_{2}$, is located near the centerline 


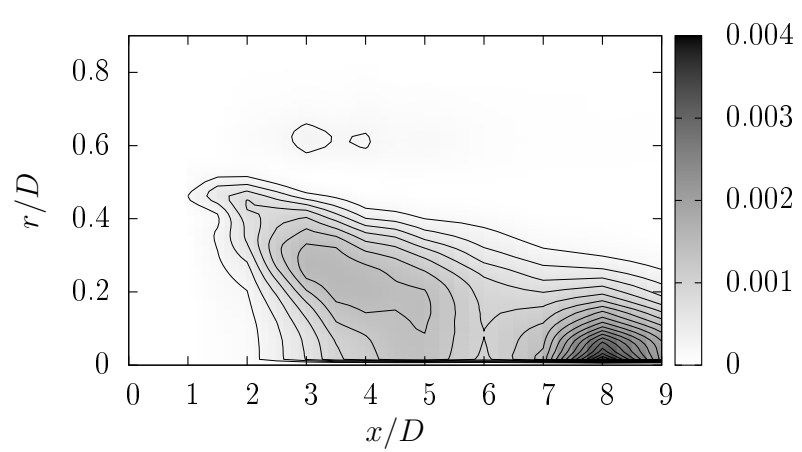

(a) $\mathcal{Y}$ axial component.

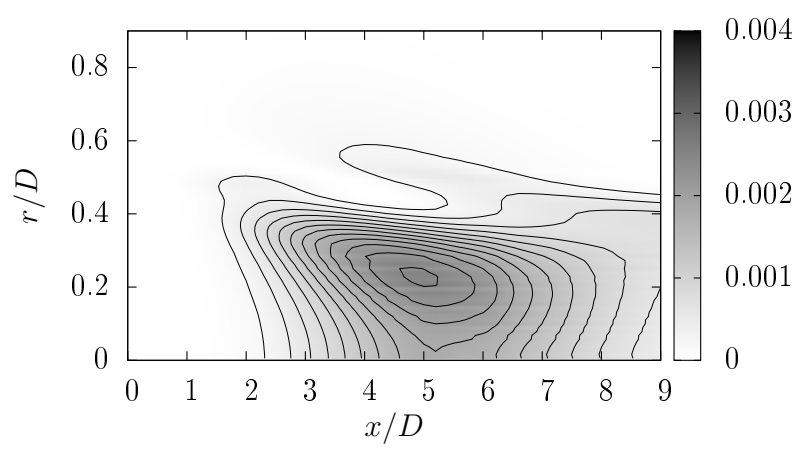

(c) PSE solution, axial component of $\left|\widetilde{\boldsymbol{q}}_{\omega, m}\right|^{2}$.

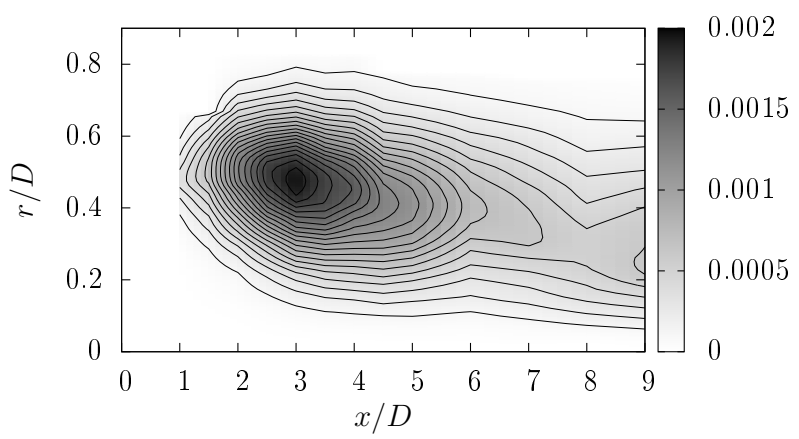

(b) $\mathcal{Y}$ radial component.

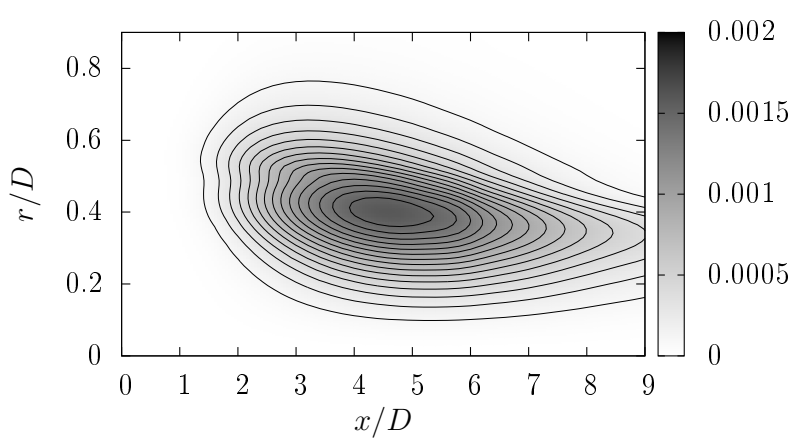

(d) PSE solution, radial component of $\left|\widetilde{\boldsymbol{q}}_{\omega, m}\right|^{2}$.

Figure 15. Comparison between observations (first POD mode of TRPIV measurements) and PSE solution.

around $x / D=5$, where the wavepacket amplitude is highest. To further explore what underlies these distinct errors of sensitivity, another computation is performed (results shown in figure 16(b)), where the domain is truncated to $x_{\max } / D=7$, such that the increase of experimental values near the centerline at $x / D=8$ is not observed. $\mathcal{S}_{2}$ disappears, and $\mathcal{S}_{1}$ remains nearly the same, showing that the small additional centerline forcing identified by $4 \mathrm{D}$-Var appears to arise in order to reduce the downstream error. The associated mechanisms are not clear for moment and require further investigation.

The high sensitivity at $\mathcal{S}_{1}$ is also predicted by the locally-parallel resolvent analysis of section III.C.3 and so it is interesting to see that the $4 \mathrm{D}$-Var also identifies this region, in the context of slowly varying mean flow, as one to exploit in order to match measurements.

In order to further characterise the forcing, the radial structures of the $4 \mathrm{D}$-Var forcing are compared with the optimal forcing modes of the resolvent analysis (section III.C), at $x / D=3$ in figure 17 (a) and at $x / D=8$ in figure $17(\mathrm{~b})$. The first location is near the maximum of the most upstream forcing spot $\mathcal{S}_{1}$. The second is more characteristic of structures downstream of the potential core. At $x / D=3$, the $4 \mathrm{D}$-Var forcing has a wide shape, similar to that of the first optimal forcing mode. The maximum is located at the critical layer and the associated OR shape corresponds qualitatively to the responses of the model problem of section III.C.2. At this point, we can postulate that the spot of sensitivity $\mathcal{S}_{1}$ figure $16($ a) is associated with a critical layer mechanism. At $x / D=8$, the forcing of $4 \mathrm{D}$-Var is three orders of magnitude weaker and shows a less clear behavior. The global shape roughly follows that of the first optimal forcing mode and has two "bumps": one around the critical layer and one near the centerline.

Let us focus on the sensitivity $\mathcal{S}_{1}$ that peaks at $x / D=3$. To understand this sensitivity, it can be noted that this axial position is the one where the wavepacket growth rate $-\operatorname{Im}(\alpha)$ becomes small (see figure 14(b)), such that the phase speed is nearly real and a critical layer is present. Similar computations have been performed for different Strouhal numbers, and the position of the maximum value of the forcing sensitivity is reported in figure 18, compared with the axial position where the wavepacket is neutrally stable, i.e. where $-\operatorname{Im}(\alpha)=0$. The maximum of sensitivity appears systematically just upstream the neutral position and follows the same trend. For a given profile, the maximum sensitivity is expected for a neutral wave. Here, the profile changes with axial position due to the jet divergence. Moving in the upstream direction, the 


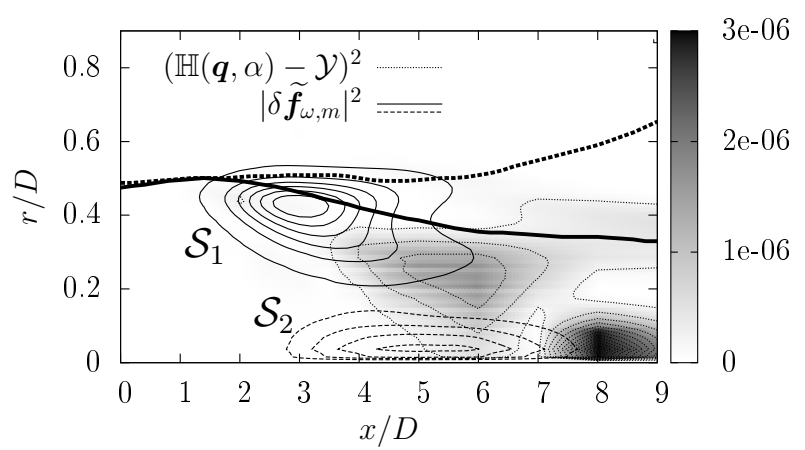

(a) $x_{\max } / D=9$.

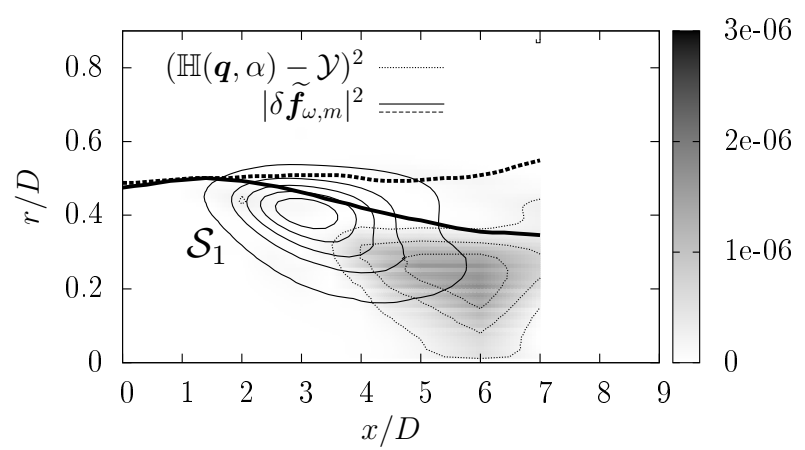

(b) $x_{\max } / D=7$.

Figure 16. Superposition of the observation error $(\mathbb{H}(\boldsymbol{q}, \alpha)-\mathcal{Y})^{2}$ (gray level and thin dotted isocontours) and the forcing sensitivity $\left|\delta \widetilde{\boldsymbol{f}}_{\omega, m}\right|^{2}$ (thin solid isocontours). Dashed lines correspond also with the forcing sensitivity $\left|\delta \widetilde{\boldsymbol{f}}_{\omega, m}\right|^{2}$, but isocontours are ten times closer from each other in order see the smaller "spot" $\mathcal{S}_{2}$. The axial component is considered. The critical layer position (thick solid line) and the inflection point position (thick dashed line) are also reported on these graphs. In figure 16(b), the final axial position $x_{\max }$ is chosen such that the errors near $x / D=8$ around the centerline are not observed. As a consequence, the near-centerline forcing $\mathcal{S}_{2}$ disappears.

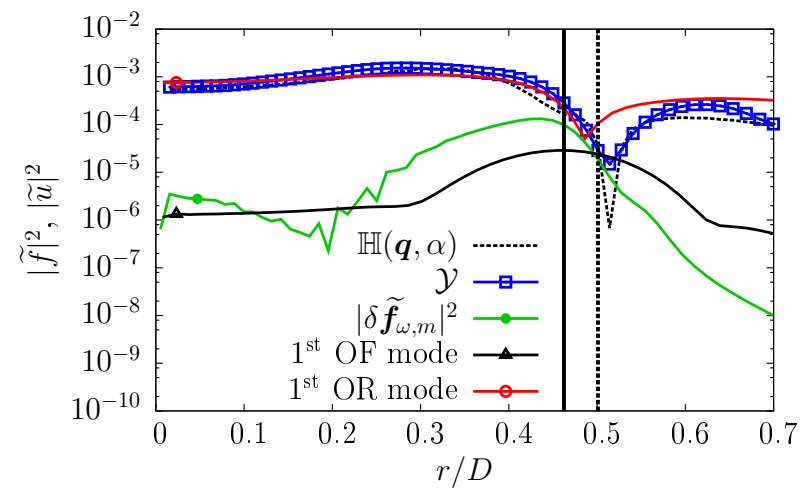

(a) $x / D=3$

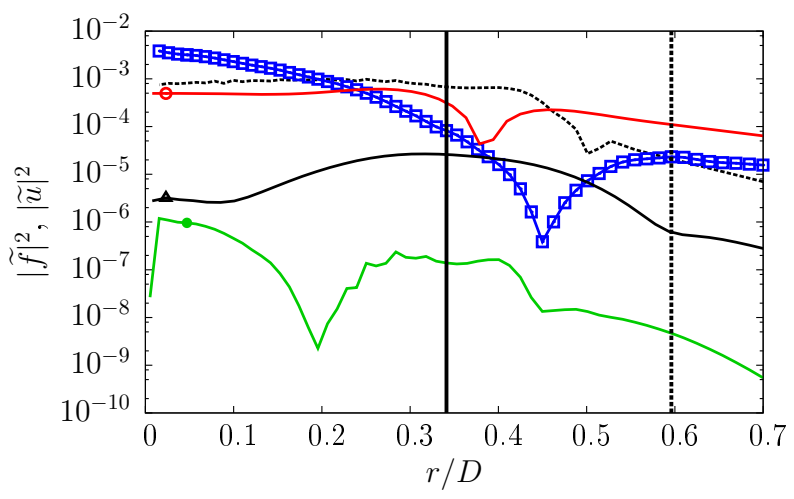

(b) $x / D=8$

Figure 17. Radial profiles of the power spectral density of the axial component. Observations $\mathcal{Y}$ (blue squares), PSE (black dashed line), forcing sensitivity $\left|\delta \widetilde{\boldsymbol{f}}_{\omega, m}\right|^{2}$ (green line), the first optimal forcing mode $\Phi_{1}$ (black line) and the associated response $\sigma_{1} \Psi_{1}$ (red line). The critical layer position (thick solid line) and the inflection point position (thick dashed line) are also reported on these graphs. 
profile becomes steeper and the critical layer and the inflection point become closer. This may explain, in accordance with section III.B, why the maximum sensitivity appears upstream of the neutral position.

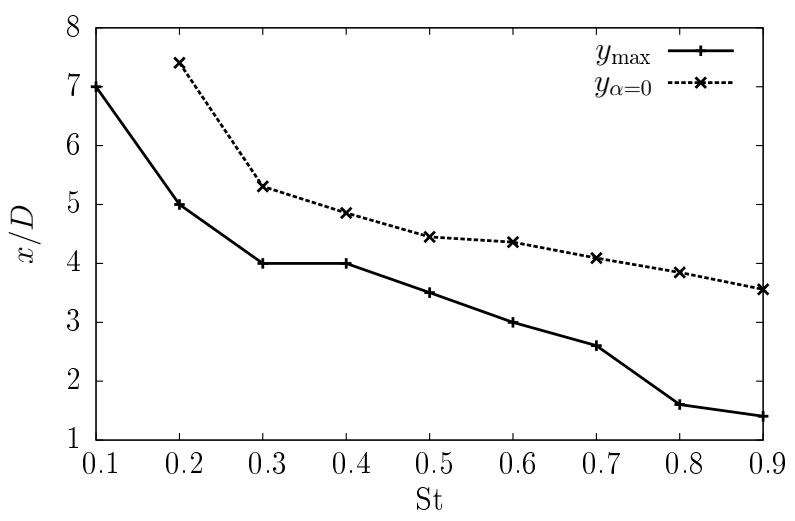

Figure 18. Axial position $y_{\max }$ of the maximum forcing sensitivity $\left|\delta \widetilde{\boldsymbol{f}}_{\omega, m}\right|^{2}$ and axial position $y_{\alpha=0}$ where the wavepacket is neutrally stable, i.e. where $-\operatorname{Im}(\alpha)=0$, function of the Strouhal number.

In order to obtain a more global sense of the relationship between forcing and response, the forcing sensitivity is superimposed, in figure 19, on the associated response $\delta \widetilde{\boldsymbol{q}}_{\omega, m}\left(\operatorname{Real}\left(\delta \widetilde{\boldsymbol{q}}_{\omega, m}^{u}\right)\right.$ figure 19(a), $\operatorname{Real}\left(\delta \widetilde{\boldsymbol{q}}_{\omega, m}^{v}\right)$ figure 19(b), $\left|\delta \widetilde{\boldsymbol{q}}_{\omega, m}^{u}\right|^{2}$ figure 19(c) and $\left|\delta \widetilde{\boldsymbol{q}}_{\omega, m}^{v}\right|^{2}$ figure 19(d)). From $x / D=1$ to $x / D=4$ (spot $\mathcal{S}_{1}$ ), it can be seen in figure 19(a) that the infinitesimal forcing and the infinitesimal response are in phase, and that both follow the critical layer and are inclined against the shear. Then, from $x / D=4$ to $x / D=9$, the forcing becomes small and the response is convected downstream. The response figures 19(c) and the error figure 16(a) can be directly compared and they correspond qualitatively. The response (figures 19(a) and 19(b)) displays traits consistent with a smooth train of vortices, centered around the critical layer: a dip of axial velocity magnitude following the critical layer in figure 19(c) and strong values of radial velocity around $(x / D \sim 6, r / D \sim 0.35)$ in figure 19(d). Figure 19(a) shows how the inclined perturbations appear to be tilted by the shear around the critical layer as well. Moreover, figure 19 (d) shows, around $r / D=0.5$, a growth and decay from $x / D \sim 6$ to $x / D \sim 9$ of the power spectral density of the radial component of the response, while the axial component remains strong and follows the critical layer. This behaviour suggests ${ }^{1}$ the presence of an Orr mechanism. It can be noted that the critical layer conditions combined with the presence of shear are necessary conditions for the Orr mechanism to operate, as pointed out by Lindzen. ${ }^{55}$ This response is consistent with the errors figure 16(a) that are strong around $x / D \sim 5-6$ with a slight error following the critical layer. The fact that the forcing sensitivity appears upstream of the associated error is due to an effect of the convective nature of the jet.

To summarise, the PSE- $4 \mathrm{D}$-Var reveals that a high sensitivity to forcing around $x / D=3$ promotes the generation of convected perturbations that decrease the error between PSE and experiment at the end of the potential core. This mechanism is consistent with the locally parallel results and is believed to be associated with the critical layer. Downstream of the potential core, results suggests that an Orr mechanism may be active around the critical layer, tilting the structures and generating a growth and decay of the power spectral density of the radial velocity. The results suggest that non-linearities may act on wavepackets via this mechanism, with significant effects in the downstream region. The high increase of error near the centerline around $x / D=8$ is for the moment not fully understood. To prevent this error, $4 \mathrm{D}$-Var finds a small additional forcing located at the end of the potential core around the centerline, but with a weak sensitivity.

\section{Conclusion}

In the present study, the effect of nonlinearities on wavepackets in jets has been considered. First, representation of the non-linear term as a harmonic "external" forcing acting on the linearised operator is found to be a useful way to generalise the standard linear wavepacket models. The response of such

\footnotetext{
${ }^{1}$ The distance of grow and decay in consistent with the distance necessary to tilt the advected perturbation predicted in Jiménez. ${ }^{54}$ Indeed, assuming a constant shear and advection velocity chosen where the maximum of $\left|\delta \widetilde{\boldsymbol{q}}_{\omega, m}^{v}\right|^{2}$ is reached, the predicted distance (based on figure $3 \mathrm{in}^{54}$ ) is $\Delta x / D \sim 2.8$, where PSE has $\Delta x / D \sim 3$.
} 


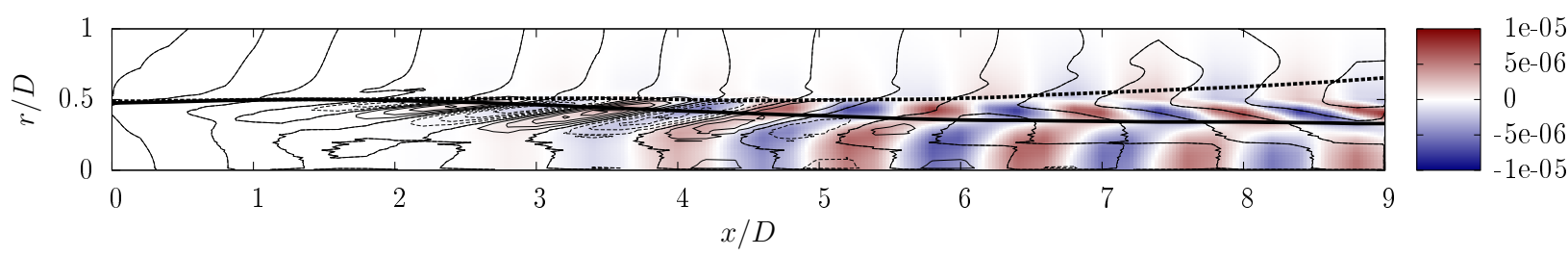

(a) Real part of the axial component. Isocontours of the forcing $\operatorname{Real}\left(\delta \widetilde{\boldsymbol{f}}_{\omega, m}^{u}\right)$ has solid lines for positive values and dashed lines for negative values.

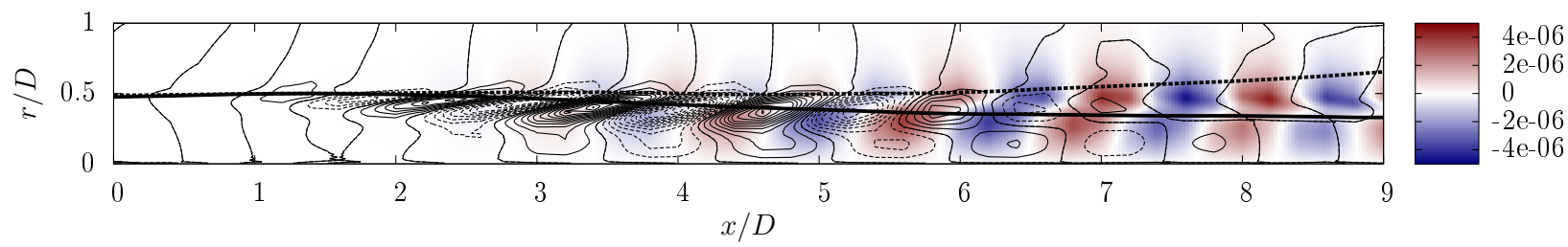

(b) Real part of the radial component. Isocontours of the forcing $\operatorname{Real}\left(\delta \widetilde{\boldsymbol{f}}_{\omega, m}^{v}\right)$ has solid lines for positive values and dashed lines for negative values.

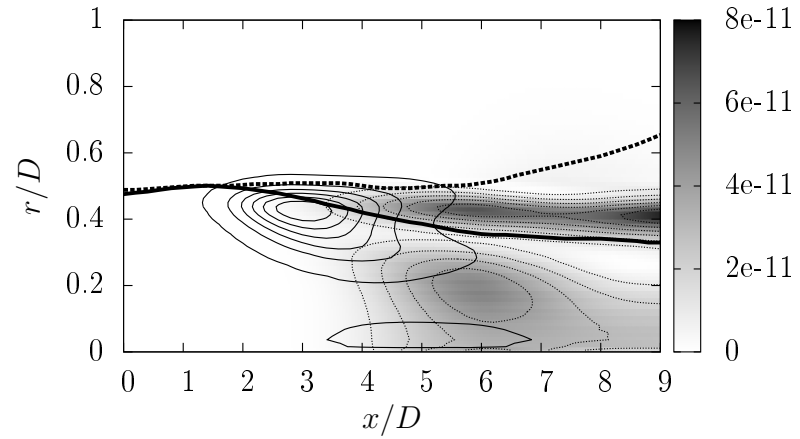

(c) Power spectral density of the axial component. Dotted lines are isoconctours of the response $\left|\delta \widetilde{\boldsymbol{q}}_{\omega, m}^{u}\right|^{2}$.

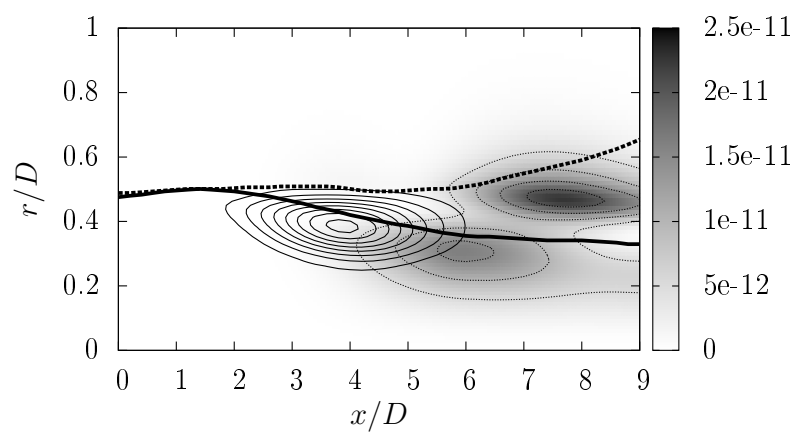

(d) Power spectral density of the radial component. Dotted lines are isoconctours of the response $\left|\delta \widetilde{\boldsymbol{q}}_{\omega, m}^{v}\right|^{2}$.

Figure 19. Superposition of the infinitesimal forcing $\delta \widetilde{\boldsymbol{f}}_{\omega, m}$ (isocontours) with the associated response $\delta \widetilde{\boldsymbol{q}}_{\omega, m}$ (colors). Real part and absolute value of the axial and radial velocity are shown from subfigures 19(a) to 19(d). The critical layer position (thick solid line) and the inflection point position (thick dashed line) are also reported on these graphs. 
models to forcing has been computed and compared with experimental data. Forced-wavepacket signatures are confirmed downstream of the potential core, where linear models fail. The sensitivity of the linear operator is systematically maximum at the critical layer, which seems to play a central role. The optimum forcing mode, predicted by a locally parallel resolvent analysis, dominates the input-output behaviour of the linearised operator and shows traits typical of a critical layer mechanism. A maximum sensitivity around $x / D=3$, upstream of the potential core is predicted by the locally parallel resolvent analysis and this is confirmed by a sensitivity analysis performed using PSE. PSE furthermore shows the effect of the convection of the response in the axial direction. One large error spot between homogeneous linear PSE and measurements downstream of the potential core has been associated with this highly sensitive zone (around $x / D=3)$. Results suggest that the response to the forcing may be driven around the critical layer by an Orr-like mechanism.

So the critical layer appears to play a central role in the forced-wavepacket scenario considered, acting in two ways. The first involves providing a high sensitivity for non-linear forcing. The second, predicted by PSE, is manifest in the tilting of perturbations. A physical explanation is that the position where the perturbation phase velocity coincides the mean flow velocity is the position where this perturbation may be transported and deformed by the shear. Both effects are due to a high non-normality of the linearised operator.

From a reduced-order modelling point of view, the optimal-forcing modes, identified in a locally parallel framework, are good candidates for the modelling of non-linearities. The optimal response mode dominates, suggesting that single-mode representations might be sufficient for modelling purposes. Before building a non-linear autonomous model, however, the following question must be answered: how do non-linearities in the jet project onto these modes? This complementary question is central for identification of the essential features necessary to predict non-linear wavepacket dynamics. It will allow the generation of a balanced basis of non-linear structures, which are both present in the jet and highly amplified by the linearised operator.

Finally, for the problem of sound radiation, the coherence decay would appear to be a key variable to match, ${ }^{5}$ if wavepacket jitter ${ }^{24}$ and quantitatively accurate sound predictions are to be obtained. Because a harmonic analysis has been considered, a perfectly coherent forcing is obtained by PSE-4D-Var and resolvent analyses. Additional post-processing will be considered in further work, in the spirit Baqui et al. ${ }^{6}$ to exploit the optimal solution for acoustic prediction.

\section{Acknowledgments}

This work was supported by the program Science Without Borders (project A073/2013), the ANR project COOLJAZZ and through fundings from the Fondation Université de Poitiers.

\section{References}

\footnotetext{
${ }^{1}$ Jordan, P. and Colonius, T., "Wave packets and turbulent jet noise," Annual Review of Fluid Mechanics, Vol. 45, 2013, pp. 173-195.

${ }^{2}$ Cavalieri, A. V. G., Jordan, P., Colonius, T., and Gervais, Y., "Axisymmetric superdirectivity in subsonic jets," Journal of Fluid Mechanics, Vol. 704, 2012, pp. 388.

${ }^{3}$ Cavalieri, A. V. G., Rodriguez, D., Jordan, P., Colonius, T., and Gervais, Y., "Wavepackets in the velocity field of turbulent jets," Journal of Fluid Mechanics, Vol. 730, 9 2013, pp. 559-592.

${ }^{4}$ Zhang, M., Jordan, P., Lehnasch, G., Cavalieri, A. V. G., and Agarwal, A., "Just enough jitter for jet noise?" $A I A A$ Aviation, American Institute of Aeronautics and Astronautics, June 2014.

${ }^{5}$ Cavalieri, A. V. G. and Agarwal, A., "Coherence decay and its impact on sound radiation by wavepackets," Journal of Fluid Mechanics, Vol. 748, 6 2014, pp. 399-415.

${ }^{6}$ Baqui, Y., Agarwal, A., and Cavalieri, A. V. G., "A coherence-matched linear model for subsonic jet noise," Proceedings of the 20th AIAA/CEAS Aeroacoustics conference, 2014.

${ }^{7}$ Breakey, D. E. S., Jordan, P., Cavalieri, A. V. G., Léon, O., Zhang, M., Lehnasch, G., Colonius, T., and Rodriguez, D., "Near-field wavepackets and the far-field sound of a subsonic jet," 19th AIAA/CEAS Aeroacoustics Conference, AIAA Paper, Vol. 2083, 2013.

${ }^{8}$ Jordan, P., Colonius, T., Brès, G. A., Zhang, M., Towne, A., and Lele, S. K., "Modeling intermittent wavepackets and their radiated sound in a turbulent jet," Proceedings of the Summer Program, Center for Turbulence Research, 2014.

${ }^{9}$ Cavalieri, A. V. G., Jordan, P., Agarwal, A., and Gervais, Y., "Jittering wave-packet models for subsonic jet noise," Journal of Sound and Vibration, Vol. 330, No. 18, 2011, pp. 4474-4492.

${ }^{10}$ Kerhervé, F., Jordan, P., Cavalieri, A. V. G., Delville, J., Bogey, C., and Juvé, D., "Educing the source mechanism associated with downstream radiation in subsonic jets," Journal of Fluid Mechanics, Vol. 710, 2012, pp. 606.

${ }^{11}$ Landahl, M. T., "A wave-guide model for turbulent shear flow," Journal of Fluid Mechanics, Vol. 29, 9 1967, pp. 441-459.
} 
${ }^{12}$ Towne, A., Colonius, T., Jordan, P., Cavalieri, A., and Brès, G., "Stochastic and nonlinear forcing of wavepackets in a Mach 0.9 jet," Abstract submitted to 21th AIAA/CEAS Aeroacoustic Conference and Exhibit, 2015, 22-26 June, Dallas, Texas.

${ }^{13}$ Maslowe, S. A., "Critical Layers in Shear Flows," Annual Review of Fluid Mechanics, Vol. 18, No. 1, 1986, pp. 405-432.

${ }^{14}$ Huerre, P. and Scott, J. F., "Effects of critical layer structure on the nonlinear evolution of waves in free shear layers," Proceedings of the Royal Society of London. A. Mathematical and Physical Sciences, Vol. 371, No. 1747, 1980, pp. 509-524.

${ }^{15}$ Huerre, P., "The nonlinear stability of a free shear layer in the viscous critical layer regime," Philosophical Transactions of the Royal Society of London. Series A, Mathematical and Physical Sciences, Vol. 293, No. 1408, 1980, pp. 643-672.

${ }^{16}$ Campos, L. M. B. C., Oliveira, J. M. G. S., and Kobayashi, M. H., "On sound propagation in a linear shear flow," Journal of Sound and Vibration, Vol. 219, No. 5, 1999, pp. 739-770.

${ }^{17}$ Haberman, R., "Nonlinear Perturbations of the Orr-Sommerfeld Equation-Asymptotic Expansion of the Logarithmic Phase Shift Across the Critical Layer," SIAM Journal on Mathematical Analysis, Vol. 7, No. 1, 1976, pp. 70-81.

${ }^{18}$ Sharma, A. S. and McKeon, B. J., "On coherent structure in wall turbulence," Journal of Fluid Mechanics, Vol. 728,8 2013, pp. 196-238.

${ }^{19}$ McKeon, B. J. and Sharma, A. S., "A critical-layer framework for turbulent pipe flow," Journal of Fluid Mechanics, Vol. 658, 9 2010, pp. 336-382.

${ }^{20}$ Dergham, G., Sipp, D., and Robinet, J.-C., "Stochastic dynamics and model reduction of amplifier flows: the backward facing step flow," Journal of Fluid Mechanics, Vol. 719, 3 2013, pp. 406-430.

${ }^{21}$ Garnaud, X., Lesshafft, L., Schmid, P. J., and Huerre, P., "The preferred mode of incompressible jets: linear frequency response analysis," Journal of Fluid Mechanics, Vol. 716, 2013, pp. 189-202.

${ }^{22}$ Nichols, J. and Jovanović, M., "Input-ouput analysis of high-speed jet noise," Proceedings of the Summer Program, Center for Turbulence Research, 2014.

${ }^{23}$ Lesshafft, L., "Preface to this Festschrift for Patrick Huerre," European Journal of Mechanics B/Fluids, Vol. 49, 2015, pp. 299-300.

${ }^{24}$ Cavalieri, A. V. G., Daviller, G., Comte, P., Jordan, P., Tadmor, G., and Gervais, Y., "Using large eddy simulation to explore sound-source mechanisms in jets," Journal of Sound and Vibration, Vol. 330, No. 17, 2011, pp. 4098-4113.

${ }^{25}$ Brès, G. A., Jordan, P., Colonius, T., Le Rallic, M., Jaunet, V., and Lele, S. K., "Large eddy simulation of a turbulent Mach 0.9 jet," Proceedings of the Summer Program, Center for Turbulence Research, 2014.

${ }^{26}$ Navon, I. M., "Data assimilation for Numerical Weather Prediction: a review," Data Assimilation for Atmospheric, Oceanic, and Hydrologic Applications, Springer Book, 2009.

${ }^{27}$ Cordier, L., Noack, B. R., Daviller, G., Tissot, G., Lehnasch, G., Delville, J., Balajewicz, M., and Niven, R., "Identification strategies for model-based control," Experiments in Fluids, Vol. 54, No. 8, 2013, pp. 1-21.

${ }^{28}$ Sasaki, K., "Estudo e controle de pacotes de onda em jatos utilizando as equaões de estabilidade parabolizadas," Master thesis, Instituto Technológico de Aeronáutica, São Josè dos Campos, Bresil, 2015.

${ }^{29}$ Gudmundsson, K. and Colonius, T., "Instability Wave Models for the Near Field Fluctuations of Turbulent Jets," Journal of Fluid Mechanics, Vol. 689, No. 97, 2011.

${ }^{30}$ Piantanida, S., Sasaki, K., Cavalieri, A. V. G., and Jordan, P., "Open-loop SISO modelling for a subsonic turbulent jet," Abstract submitted to 21th AIAA/CEAS Aeroacoustic Conference and Exhibit, 2015, 22-26 June, Dallas, Texas.

${ }^{31}$ Michalke, A., "Survey on jet instability theory," Progress in Aerospace Sciences, Vol. 21, 1984, pp. 159-199.

${ }^{32}$ Moarref, R., Jovanović, M. R., Tropp, J. A., Sharma, A. S., and McKeon, B. J., "A low-order decomposition of turbulent channel flow via resolvent analysis and convex optimization," Physics of Fluids (1994-present), Vol. 26, No. 5, 2014 , pp. 051701.

${ }^{33}$ Maslowe, S. A., "Critical Layers in Shear Flows," Lecture notes: Department of Mathematics and Statistics, McGill University, Montreal, Canada, 2009.

${ }^{34}$ Schmid, P. J. and Henningson, D. S., Stability and transition in shear flows, Vol. 142, Springer Science \& Business Media, 2001.

${ }^{35}$ Schmid, P. J., "Nonmodal stability theory," Annual Review of Fluid Mechanics, Vol. 39, 2007, pp. 129-162.

${ }^{36}$ Freund, J. B., Compressibility effects in a turbulent annular mixing layer, Ph.D. thesis, Stanford University, 1997.

${ }^{37}$ Gudmundsson, K., Instability wave models of turbulent jets from round and serrated nozzles, Ph.D. thesis, California Institute of Technology, Pasadena, California, 2010.

${ }^{38}$ Poinsot, T. J. and Lele, S. K., "Boundary conditions for direct simulations of compressible viscous flows," Journal of Computational Physics, Vol. 101, No. 1, 1992, pp. 104-129.

${ }^{39}$ Hanifi, A., Schmid, P. J., and Henningson, D. S., "Transient growth in compressible boundary layer flow," Physics of Fluids (1994-present), Vol. 8, No. 3, 1996, pp. 826-837.

${ }^{40}$ Gloor, M., Obrist, D., and Kleiser, L., "Linear stability and acoustic characteristics of compressible, viscous, subsonic coaxial jet flow," Physics of Fluids (1994-present), Vol. 25, No. 8, 2013, pp. 084102.

${ }^{41}$ Weideman, J. A. and Reddy, S. C., "A MATLAB Differentiation Matrix Suite," ACM Trans. Math. Softw., Vol. 26, No. 4, Dec. 2000, pp. 465-519.

${ }^{42}$ Rowley, C. W., Colonius, T., and Murray, R. M., "Model reduction for compressible flows using POD and Galerkin projection," Physica D: Nonlinear Phenomena, Vol. 189, No. 1-2, 2004, pp. 115-129.

${ }^{43}$ Farrell, B. F. and Ioannou, P. J., "Generalized Stability Theory. Part I: Autonomous Operators," Journal of the Atmospheric Sciences, Vol. 53, No. 14, July 1996, pp. 2025-2040.

${ }^{44}$ Papadakis, N., Assimilation de données images : application au suivi de courbes et de champs de vecteurs, $\mathrm{Ph} . \mathrm{D}$. thesis, Université de Rennes I, 2007.

${ }^{45}$ Herbert, T., "Parabolized stability equations," Annual Review of Fluid Mechanics, Vol. 29, No. 1, 1997, pp. $245-283$.

${ }^{46}$ Crighton, D. G. and Gaster, M., "Stability of slowly diverging jet flow," Journal of Fluid Mechanics, Vol. 77, No. 2, 1976, pp. 397-413. 
${ }^{47}$ Cordier, L., "Flow control and constrained optimization problems," Reduced-Order Modelling for Flow Control, Vol. 528 of CISM Courses and Lectures, Springer Vienna, 2011, pp. 1-76.

${ }^{48}$ Dobrinsky, A., Adjoint analysis for receptivity prediction, Ph.D. thesis, Rice University, Houston, Texas, 2002.

${ }^{49}$ Pralits, J. O., Airiau, C., Hanifi, A., and Henningson, D. S., "Sensitivity analysis using adjoint parabolized stability equations for compressible flows," Flow, turbulence and combustion, Vol. 65, No. 3-4, 2000, pp. 321-346.

${ }^{50}$ Pralits, J. O., Hanifi, A., and Henningson, D. S., "Adjoint-based optimization of steady suction for disturbance control in incompressible flows," Journal of Fluid Mechanics, Vol. 467, 2002, pp. 129-161.

${ }^{51}$ Airiau, C., Bottaro, A., Walther, S., and Legendre, D., "A methodology for optimal laminar flow control: Application to the damping of Tollmien-Schlichting waves in a boundary layer," Physics of Fluids (1994-present), Vol. 15, No. 5, 2003, pp. 1131-1145.

${ }^{52}$ Bewley, T. R., Moin, P., and Temam, R., "DNS-based predictive control of turbulence: an optimal benchmark for feedback algorithms," Journal of Fluid Mechanics, Vol. 447, 10 2001, pp. 179-225.

${ }^{53}$ Nocedal, J. and Wright, S. J., Numerical optimization, Springer, 1999.

${ }^{54}$ Jiménez, J., "How linear is wall-bounded turbulence?" Physics of Fluids, Vol. 25, 2013, pp. 110814.

${ }^{55}$ Lindzen, R. S., "Instability of plane parallel shear flow (toward a mechanistic picture of how it works)," pure and applied geophysics, Vol. 126, No. 1, 1988, pp. 103-121.

\section{Appendix}

\section{Full set of equations used in the Resolvent analysis}

In the appendix, we present the full governing equation for the linearised Navier-Stokes system used for the resolvent analysis. The equations are written in terms of $(\widetilde{u}, \widetilde{v}, \widetilde{\rho}, \widetilde{T})$ in cylindrical coordinate.

GOVERNING EQUATION FOR $x$-MOMENTUM

$$
\begin{aligned}
-i \omega \widetilde{u}+i \alpha \bar{U} \widetilde{u} & +\widetilde{v} \bar{U}^{\prime}=-\frac{\gamma-1}{\gamma} i \alpha \widetilde{T}-\frac{\gamma-1}{\gamma} \frac{\bar{T}}{\bar{\rho}} i \alpha \widetilde{\rho} \\
+ & \frac{1}{\bar{\rho} R e}\left[\bar{\mu}\left(-\alpha^{2} \widetilde{u}+\frac{1}{r} \frac{\partial}{\partial r}\left(r \frac{\partial \widetilde{u}}{\partial r}\right)-\frac{m^{2}}{r^{2}} \widetilde{u}\right)+\frac{\bar{\mu}}{3}\left(-\alpha^{2} \widetilde{u}+i \alpha \frac{\partial \widetilde{v}}{\partial r}+\frac{i \alpha}{r} \widetilde{v}-\frac{\alpha m}{r} \widetilde{w}\right)\right. \\
& \left.+\frac{\partial \bar{\mu}}{\partial r}\left(i \alpha \widetilde{v}+\frac{\partial \widetilde{u}}{\partial r}\right)+\frac{\mu}{r} \frac{\partial}{\partial r}\left(r \frac{\partial \bar{U}}{\partial r}\right)+\frac{\partial \mu}{\partial r} \frac{\partial \bar{U}}{\partial r}+\bar{\mu}_{B}\left(-\alpha^{2} \widetilde{u}+i \alpha \frac{\partial \widetilde{v}}{\partial r}+\frac{i \alpha}{r} \widetilde{v}-\frac{\alpha m}{r} \widetilde{w}\right)\right]
\end{aligned}
$$

\section{GOVERNING EQUATION FOR $r$-MOMENTUM}

$$
\begin{aligned}
&-i \omega \widetilde{v}+i \alpha \bar{U} \widetilde{v}=-\frac{\gamma-1}{\gamma} \frac{\partial \widetilde{T}}{\partial r}-\frac{\gamma-1}{\gamma} \frac{\bar{T}}{\bar{\rho}} \frac{\partial \widetilde{\rho}}{\partial r}-\frac{\gamma-1}{\gamma} \frac{\tilde{\rho}}{\bar{\rho}} \frac{\partial \bar{T}}{\partial r}-\frac{\gamma-1}{\gamma} \frac{\widetilde{T}}{\bar{\rho}} \frac{\partial \bar{\rho}}{\partial r} \\
&+ \frac{1}{\bar{\rho} R e}\left[\bar{\mu}\left(-\alpha^{2} \widetilde{v}+\frac{1}{r} \frac{\partial}{\partial r}\left(r \frac{\partial \widetilde{v}}{\partial r}\right)-\frac{m^{2}}{r^{2}} \widetilde{v}\right)+\frac{\bar{\mu}}{3}\left(i \alpha \frac{\partial \widetilde{u}}{\partial r}+\frac{\partial^{2} \widetilde{v}}{\partial r^{2}}+\frac{1}{r} \frac{\partial \widetilde{v}}{\partial r}-\frac{\widetilde{v}}{r^{2}}+\frac{i m}{r} \frac{\partial \widetilde{w}}{\partial r}-\frac{i m}{r^{2}} \widetilde{w}\right)\right. \\
&-\frac{\bar{\mu} \widetilde{v}}{r^{2}}-\frac{2 i m \bar{\mu}}{r^{2}} \widetilde{w}+\frac{\partial \bar{\mu}}{\partial r}\left(2 \frac{\partial \widetilde{v}}{\partial r}-\frac{2 i \alpha}{3} \widetilde{u}-\frac{2}{3} \frac{\partial \widetilde{v}}{\partial r}-\frac{2}{3} \frac{\tilde{v}}{r}-\frac{2}{3} \frac{i m}{r} \widetilde{w}\right)+i \alpha \mu \bar{U}^{\prime} \\
&\left.+\bar{\mu}_{B}\left(i \alpha \frac{\partial \widetilde{u}}{\partial r}+\frac{\partial^{2} \widetilde{v}}{\partial r^{2}}+\frac{3}{r} \frac{\partial \widetilde{v}}{\partial r}+\frac{\widetilde{v}}{r^{2}}+\frac{i m}{r} \frac{\partial \widetilde{w}}{\partial r}+\frac{i m}{r^{2}} \widetilde{w}+\frac{2 i \alpha}{r} \widetilde{u}\right)+\frac{\partial \bar{\mu}_{B}}{\partial r}\left(i \alpha \widetilde{u}+\frac{\partial \widetilde{v}}{\partial r}+\frac{\widetilde{v}}{r}+\frac{i m}{r} \widetilde{w}\right)\right]
\end{aligned}
$$

Continuity EQUATION

$$
-i \omega \widetilde{\rho}+i \alpha \bar{U} \widetilde{\rho}+\bar{\rho} i \alpha \widetilde{u}+\bar{\rho} \frac{\partial \widetilde{v}}{\partial r}+\widetilde{v} \frac{\partial \bar{\rho}}{\partial r}+\frac{\bar{\rho} \widetilde{v}}{r}+\frac{i m \bar{\rho}}{r} \widetilde{w}=0
$$

\section{ENERGY EQUATION}

$$
\begin{aligned}
-i \omega \widetilde{T}+\widetilde{v} \frac{\partial \bar{T}}{\partial r}+i \alpha \bar{U} \widetilde{T}+(\gamma-1) \bar{T}\left(i \alpha \widetilde{u}+\frac{\partial \widetilde{v}}{\partial r}+\frac{\widetilde{v}}{r}+\frac{i m}{r} \widetilde{w}\right)= \\
\frac{\gamma}{\bar{\rho} \operatorname{RePr}}\left[\bar{\mu}\left(-\alpha^{2} \widetilde{T}+\frac{\partial^{2} \widetilde{T}}{\partial r^{2}}+\frac{1}{r} \frac{\partial \widetilde{T}}{\partial r}-\frac{m^{2}}{r^{2}} \widetilde{T}\right)+\mu\left(\frac{\partial^{2} \bar{T}}{\partial r^{2}}+\frac{1}{r} \frac{\partial \bar{T}}{\partial r}\right)+\frac{\partial \bar{\mu}}{\partial r} \frac{\partial \widetilde{T}}{\partial r}+\frac{\partial \mu}{\partial r} \frac{\partial \bar{T}}{\partial r}\right] \\
+\frac{\gamma}{\bar{\rho}}\left[\frac{2 \bar{\mu}}{R e} \frac{\partial \bar{U}}{\partial r}\left(i \alpha \widetilde{v}+\frac{\partial \widetilde{u}}{\partial r}\right)+\frac{\mu}{R e}\left(\frac{\partial \bar{U}}{\partial r}\right)^{2}\right]
\end{aligned}
$$




\section{Complementary results of the Resolvent analysis}

In this appendix, a complete view of the resolvent analysis is given. Figure 20 compares for a range of axial positions $x=[2-9]$ the power spectral density of the axial velocity fluctuation measured by TRPIV, predicted by PSE and of the optimal response modes. The corresponding phases are showed figure 21 .

As mentioned in the text, PSE and LEE begin to get away from measurements at $x / D=5$, while optimal response continue to have a dip close to the experimental one. The optimal forcing has systematically a maximum at the critical layer. The phase jump, shown in figure 21, is systematically a $\pi$ jump, sign of a vortex-like structure. Experiments and PSE predict a smooth change of the phase, while resolvent analysis predicts a sharp jump.

\section{Derivation of gradient of PSE-4D-Var}

In order to obtain the optimality system (29), (30) and (31), we have to derive the Lagrangian (26)-(28) with respect to each variable. We note $\nabla_{\phi} \mathcal{L}$ the gradient of $\mathcal{L}$ respect to a variable $\phi$, defined such that for any variation $\delta \phi$, we have

$$
\left(\nabla_{\phi} \mathcal{L}, \delta \phi\right)_{\phi}=\lim _{\epsilon \rightarrow 0} \frac{\mathcal{L}(\phi+\epsilon \delta \phi)-\mathcal{L}(\phi)}{\epsilon} .
$$

The inner product $(\cdot, \cdot)_{\phi}$ is the inner product in the space where $\phi$ lies. For the PSE, we define three different inner products:

$$
\begin{aligned}
(a, b)_{x} & =\int_{0}^{L} a(x) b(x) \mathrm{d} x \\
(a, b)_{r} & =\int_{0}^{\infty} a(r) b(r) r \mathrm{~d} r \\
(a, b)_{x r} & =\int_{0}^{L} \int_{0}^{\infty} a(x, r) b(x, r) r \mathrm{~d} r \mathrm{~d} x=\int_{0}^{L}(a, b)_{r} \mathrm{~d} x
\end{aligned}
$$

Directional derivative in the directions $\delta \boldsymbol{\lambda}$ and $\delta \zeta$

Using the definition (39), we obtain

$$
\begin{aligned}
\left(\nabla_{\boldsymbol{\lambda}} \mathcal{L}, \delta \boldsymbol{\lambda}\right)_{x r} & =-\operatorname{Real}\left(\int_{0}^{L}\left(E \frac{\partial \boldsymbol{q}}{\partial x}+(A+\alpha B) \boldsymbol{q}-\boldsymbol{w}, \delta \boldsymbol{\lambda}\right)_{r} \mathrm{~d} x\right) \\
\left(\nabla_{\zeta} \mathcal{L}, \delta \zeta\right)_{x} & =-\operatorname{Real}\left(\int_{0}^{L}\left(\boldsymbol{q}, \frac{\partial \boldsymbol{q}}{\partial x}\right)_{r} \delta \zeta \mathrm{d} x\right) .
\end{aligned}
$$

This set of equations means that when the minimum is reached, the constraints (21) are respected. Indeed, at the minimum we have $\nabla_{\lambda} \mathcal{L}=\nabla_{\zeta} \mathcal{L}=0$ for each $\delta \boldsymbol{\lambda}$, and $\delta \zeta$. The direct system (29) is then a part of the optimality system. As a consequence, when the minimum is reached, we also have $\mathcal{J}=\mathcal{L}$.

\section{Directional derivative in the directions $\delta \boldsymbol{q}$}

Because the derivation is long, we will treat the different terms (26) to (28) separately.

TERM (26):

$$
\begin{aligned}
\left(\nabla_{\boldsymbol{q}} \mathcal{L}, \delta \boldsymbol{q}\right)_{x r}^{(26)}= & \lim _{\epsilon \rightarrow 0} \frac{1}{\epsilon} \operatorname{Real}\left(\int_{0}^{L}\left(Q\left(|\boldsymbol{q}+\epsilon \delta \boldsymbol{q}|^{2}-|\boldsymbol{q}|^{2}\right) \mid e^{\left.i \int_{0}^{x} \alpha(\xi) \mathrm{d} \xi\right|^{2}}, \mathbb{H}(\boldsymbol{q}, \alpha)-\mathcal{Y}\right)_{W_{o}} \mathrm{~d} x\right) \\
& +\lim _{\epsilon \rightarrow 0} \frac{1}{\epsilon} \operatorname{Real}\left(\left(Q\left(\left|\boldsymbol{q}_{L}+\epsilon \delta \boldsymbol{q}_{L}\right|^{2}-\left|\boldsymbol{q}_{L}\right|^{2}\right)\left|e^{i \int_{0}^{L} \alpha(\xi) \mathrm{d} \xi}\right|^{2}, \mathbb{H}\left(\boldsymbol{q}_{L}, \alpha_{L}\right)-\mathcal{Y}_{L}\right)_{W_{T}}\right) \\
= & \operatorname{Real}\left(\int_{0}^{L}\left(\delta \boldsymbol{q}, 2 \mid e^{\left.i \int_{0}^{x} \alpha(\xi) \mathrm{d} \xi\right|^{2}}\left(\left(Q^{*} Q D_{\overline{\boldsymbol{q}}}\right)^{+} Q^{*} W_{o}(\mathbb{H}(\boldsymbol{q}, \alpha)-\mathcal{Y})\right)\right)_{r} \mathrm{~d} x\right) \\
& +\operatorname{Real}\left(\left(\delta \boldsymbol{q}_{L}, 2\left|e^{i \int_{0}^{L} \alpha(\xi) \mathrm{d} \xi}\right|^{2}\left(\left(Q^{*} Q D_{\overline{\boldsymbol{q}}}\right)^{+} Q^{*} W_{T}\left(\mathbb{H}\left(\boldsymbol{q}_{L}, \alpha_{L}\right)-\mathcal{Y}_{L}\right)\right)\right)_{r}\right)
\end{aligned}
$$



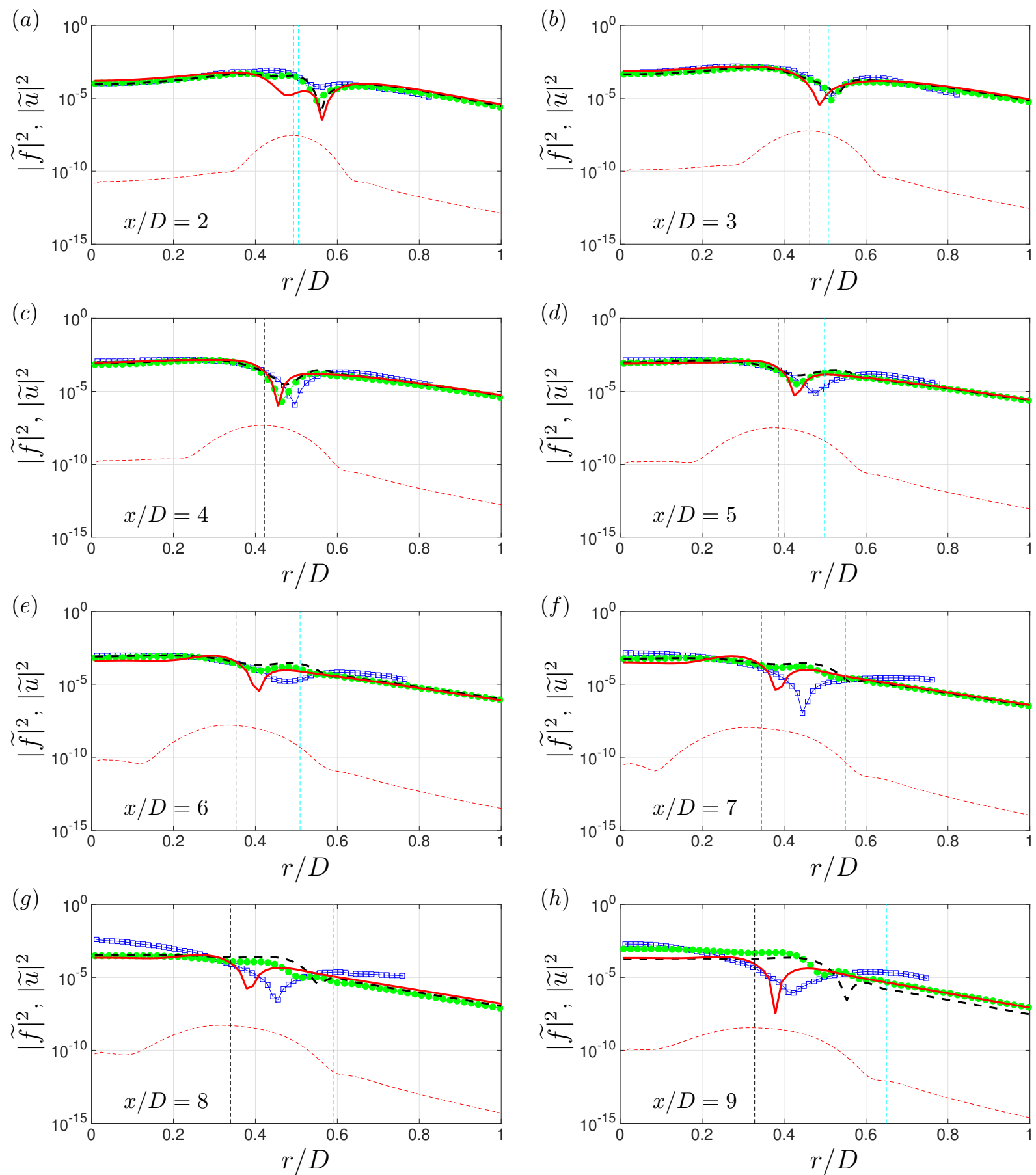

Figure 20. Comparison of optimal forcing (red dashed lines) and the optimal response modes (red solid lines) of axial velocity fluctuation at $x / D=2 \sim 9$ with experimental data (blue squares) and the solution of the homogeneous linear problem (LEE: green line with solid dots ; PSE: black dashed line). St $=0.6, R e=10000, M=0.4 . \quad c$ is determined from the PSE calculation. The vertical dashed line is the critical layer position and the vertical dashed cyan line the inflectional point position. 

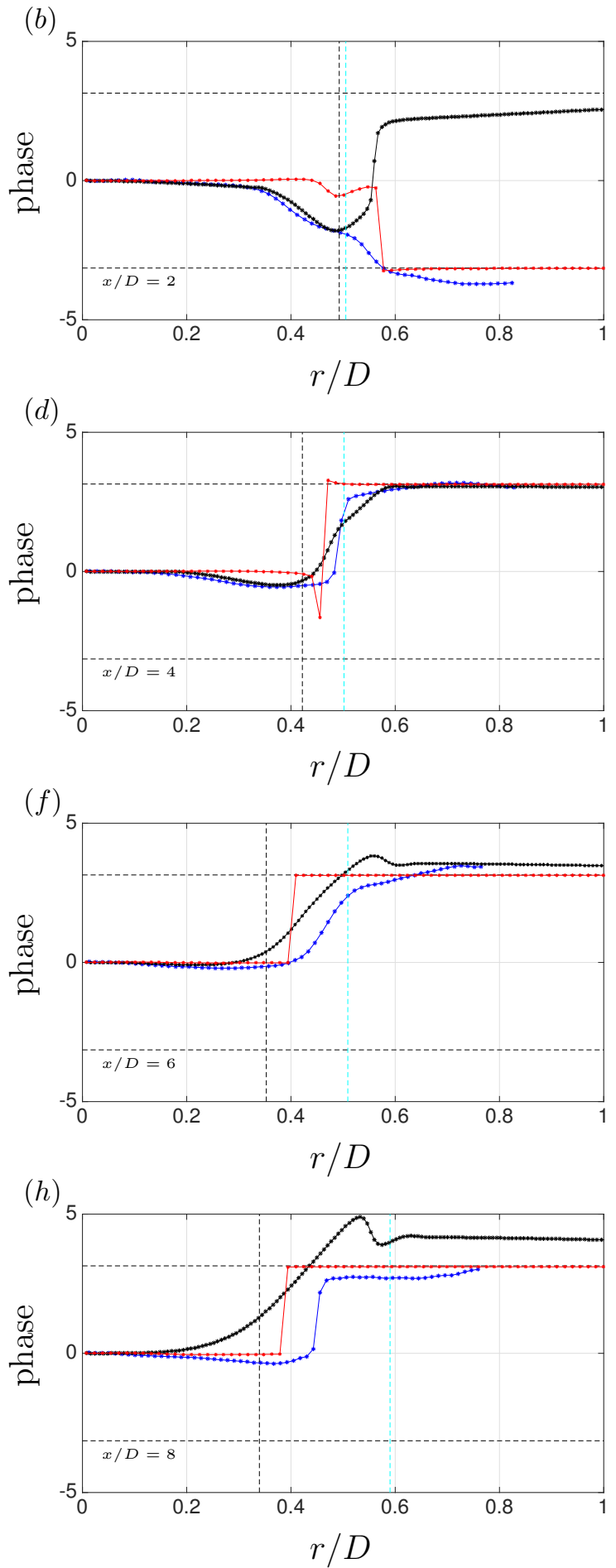
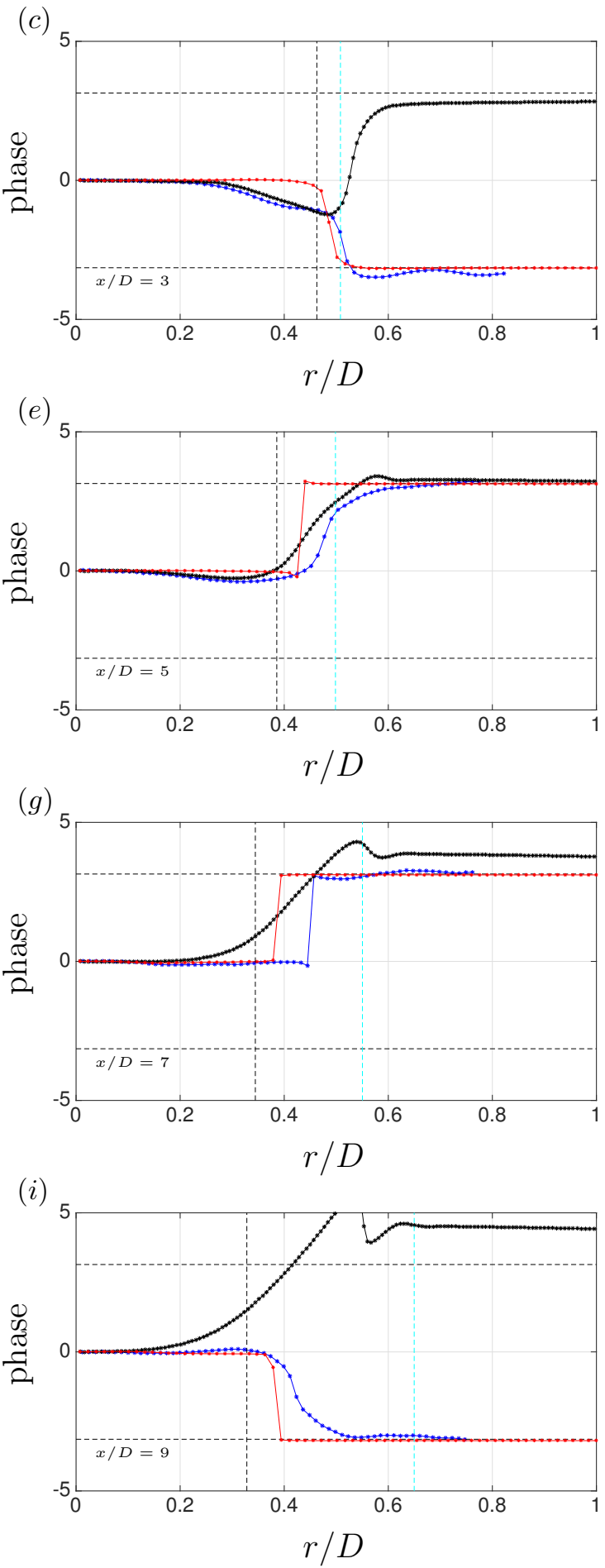

Figure 21. Comparison of the shifted phases of axial velocity fluctuation at $x / D=2 \sim 9$. OR of RA: red line); TRPIV: blue line; PSE: black line. The phases are shifted to have zero reference phase at $r / D=0$. St $=0.6, R e=10000, M=0.4$ and PSE-determined $c$. The two horizontal dashed lines are at $-\pi$ and $\pi$. The vertical dashed lines are the critical layer position (according to the PSE solution) and the vertical dashed cyan line the inflectional point position. 
where $D_{\overline{\boldsymbol{q}}}=\operatorname{diag}(\overline{\boldsymbol{q}})$.

TERM (27):

$$
\begin{aligned}
\left(\nabla_{\boldsymbol{q}} \mathcal{L}, \delta \boldsymbol{q}\right)_{x r}^{(27)}= & -\operatorname{Real}\left(\int_{0}^{L}\left(E \frac{\partial \delta \boldsymbol{q}}{\partial x}+(A+\alpha B) \delta \boldsymbol{q}, \boldsymbol{\lambda}\right)_{r} \mathrm{~d} x\right) \\
= & -\operatorname{Real}\left(\int_{0}^{L}\left(\delta \boldsymbol{q},-E^{+} \frac{\partial \boldsymbol{\lambda}}{\partial x}+\left(A+\alpha B-\frac{\partial E}{\partial x}\right)^{+} \boldsymbol{\lambda}\right)_{r} \mathrm{~d} x\right) \\
& -\operatorname{Real}\left(\left[\left(\delta \boldsymbol{q}, E^{+} \boldsymbol{\lambda}\right)_{r}\right]_{0}^{L}\right)
\end{aligned}
$$

The treatment of this term reveals the terminal condition $\boldsymbol{\lambda}(L)=0$ equation (46b). Because the initial condition is implicitly enforced, we have $\delta \boldsymbol{q}(0, r)=0$. No boundary condition appears explicitly, but because the discrete adjoint in the radial direction is used, symmetries at $r / D=0$ and Dirichlet boundary condition at $r / D \rightarrow \infty$ are implicitly enforced.

TERM (28):

$$
\begin{aligned}
\left(\nabla_{\boldsymbol{q}} \mathcal{L}, \delta \boldsymbol{q}\right)_{x r}^{(28)}= & -\operatorname{Real}\left(\int_{0}^{L}\left(\delta \boldsymbol{q}, \frac{\partial \boldsymbol{q}}{\partial x}\right)_{r} \zeta+\left(\boldsymbol{q}, \frac{\partial \delta \boldsymbol{q}}{\partial x}\right)_{r} \zeta \mathrm{d} x\right) \\
= & -\operatorname{Real}\left(\int_{0}^{L}\left(\delta \boldsymbol{q}, \frac{\partial \boldsymbol{q}}{\partial x}\left(\zeta-\zeta^{*}\right)-\boldsymbol{q} \frac{\partial \zeta^{*}}{\partial x}\right)_{r} \mathrm{~d} x\right) \\
& -\operatorname{Real}\left(\left[(\delta \boldsymbol{q}, \boldsymbol{q})_{r} \zeta^{*}\right]_{0}^{L}\right)
\end{aligned}
$$

Equation (47b) gives the terminal condition $\zeta(L)=0$.

Directional derivative in the directions $\delta \alpha$

Here, the variations of $\mathcal{L}$ respect to the real and imaginary part of $\alpha=\alpha_{r}+i \alpha_{i}$ are considered separately.

$$
\begin{aligned}
\left(\nabla_{\alpha_{r}} \mathcal{L}, \delta \alpha_{r}\right)_{x}= & \lim _{\epsilon \rightarrow 0} \frac{1}{\epsilon} \operatorname{Real}\left(\int_{0}^{L}\left(Q\left|\boldsymbol{q} e^{i \int_{0}^{x} \alpha(\xi)+\epsilon \delta \alpha_{r}(\xi) \mathrm{d} \xi}\right|^{2}-Q\left|\boldsymbol{q} e^{i \int_{0}^{x} \alpha(\xi) \mathrm{d} \xi}\right|^{2}, \mathbb{H}(\boldsymbol{q}, \alpha)-\mathcal{Y}\right)_{W_{o}} \mathrm{~d} x\right) \\
& +\lim _{\epsilon \rightarrow 0} \frac{1}{\epsilon} \operatorname{Real}\left(\left(Q\left|\boldsymbol{q}_{L} e^{i \int_{0}^{L} \alpha(\xi)+\epsilon \delta \alpha_{r}(\xi) \mathrm{d} \xi}\right|^{2}-Q\left|\boldsymbol{q}_{L} e^{i \int_{0}^{L} \alpha(\xi) \mathrm{d} \xi}\right|^{2}, \mathbb{H}\left(\boldsymbol{q}_{L}, \alpha_{L}\right)-\mathcal{Y}_{L}\right)_{W_{T}}\right) \\
& -\operatorname{Real}\left(\int_{0}^{L}\left(\delta \alpha_{r} B \boldsymbol{q}, \boldsymbol{\lambda}\right)_{r} \mathrm{~d} x\right) \\
= & -\operatorname{Real}\left(\int_{0}^{L} \delta \alpha_{r}(B \boldsymbol{q}, \boldsymbol{\lambda})_{r} \mathrm{~d} x\right)
\end{aligned}
$$




$$
\begin{aligned}
\left(\nabla_{\alpha_{i}} \mathcal{L}, \delta \alpha_{i}\right)_{x}= & \lim _{\epsilon \rightarrow 0} \frac{1}{\epsilon} \operatorname{Real}\left(\int_{0}^{L}\left(Q\left|\boldsymbol{q} e^{i \int_{0}^{x} \alpha(\xi)+i \epsilon \delta \alpha_{i}(\xi) \mathrm{d} \xi}\right|^{2}-Q\left|\boldsymbol{q} e^{i \int_{0}^{x} \alpha(\xi) \mathrm{d} \xi}\right|^{2}, \mathbb{H}(\boldsymbol{q}, \alpha)-\mathcal{Y}\right)_{W_{o}} \mathrm{~d} x\right) \\
& +\lim _{\epsilon \rightarrow 0} \frac{1}{\epsilon} \operatorname{Real}\left(\left(Q\left|\boldsymbol{q}_{L} e^{i \int_{0}^{L} \alpha(\xi)+i \epsilon \delta \alpha_{i}(\xi) \mathrm{d} \xi}\right|^{2}-Q\left|\boldsymbol{q}_{L} e^{i \int_{0}^{L} \alpha(\xi) \mathrm{d} \xi}\right|^{2}, \mathbb{H}\left(\boldsymbol{q}_{L}, \alpha_{L}\right)-\mathcal{Y}_{L}\right)_{W_{T}}\right) \\
& -\operatorname{Real}\left(\int_{0}^{L}\left(i \delta \alpha_{i} B \boldsymbol{q}, \boldsymbol{\lambda}\right)_{r} \mathrm{~d} x\right) \\
= & -2 \int_{0}^{L} \int_{0}^{x} \delta \alpha_{i} \mathrm{~d} \xi(\mathbb{H}(\boldsymbol{q}, \alpha), \mathbb{H}(\boldsymbol{q}, \alpha)-\mathcal{Y})_{W_{o}} \mathrm{~d} x \\
& -2 \int_{0}^{L} \delta \alpha_{i} \mathrm{~d} \xi\left(\mathbb{H}\left(\boldsymbol{q}_{L}, \alpha_{L}\right), \mathbb{H}\left(\boldsymbol{q}_{L}, \alpha_{L}\right)-\mathcal{Y}_{L}\right)_{W_{T}}-\operatorname{Im}\left(\int_{0}^{L} \delta \alpha_{i}(B \boldsymbol{q}, \boldsymbol{\lambda})_{r} \mathrm{~d} x\right) \\
= & -2 \int_{0}^{L} \delta \alpha_{i} \int_{x}^{L}(\mathbb{H}(\boldsymbol{q}, \alpha), \mathbb{H}(\boldsymbol{q}, \alpha)-\mathcal{Y})_{W_{o}} \mathrm{~d} \xi \mathrm{d} x \\
& -2 \int_{0}^{L} \delta \alpha_{i}\left(\mathbb{H}\left(\boldsymbol{q}_{L}, \alpha_{L}\right), \mathbb{H}\left(\boldsymbol{q}_{L}, \alpha_{L}\right)-\mathcal{Y}_{L}\right)_{W_{T}} \mathrm{~d} \xi-\operatorname{Im}\left(\int_{0}^{L} \delta \alpha_{i}(B \boldsymbol{q}, \boldsymbol{\lambda})_{r} \mathrm{~d} x\right)
\end{aligned}
$$

Details of calculation of the adjoint of the cumulative integral are present later in the appendix. We finally have

$$
\begin{aligned}
& \left(\nabla_{\alpha} \mathcal{L}, \delta \alpha\right)_{x}= \\
& \operatorname{Real}\left(\int_{0}^{L} \delta \alpha\left(-2 i \int_{x}^{L}(\mathbb{H}(\boldsymbol{q}, \alpha), \mathbb{H}(\alpha)-\mathcal{Y})_{W_{o}} \mathrm{~d} \xi-2 i\left(\mathbb{H}\left(\boldsymbol{q}_{L}, \alpha_{L}\right), \mathbb{H}\left(\alpha_{L}\right)-\mathcal{Y}_{L}\right)_{W_{T}}-(B \boldsymbol{q}, \boldsymbol{\lambda})_{r}\right) \mathrm{d} x\right)
\end{aligned}
$$

Directional derivative in the directions $\delta \boldsymbol{w}$

$$
\begin{aligned}
\left(\nabla_{\boldsymbol{w}} \mathcal{L}, \delta \boldsymbol{w}\right)_{x r} & =\operatorname{Real}\left(\int_{0}^{L}(\delta \boldsymbol{w}, \boldsymbol{w})_{W_{w}} \mathrm{~d} x\right)-\operatorname{Real}\left(\int_{0}^{L}(-\delta \boldsymbol{w}, \boldsymbol{\lambda})_{r} \mathrm{~d} x\right) \\
& =\operatorname{Real}\left(\int_{0}^{L}\left(\delta \boldsymbol{w}, W_{w} \boldsymbol{w}+\boldsymbol{\lambda}\right)_{r} \mathrm{~d} x\right)
\end{aligned}
$$

\section{Assembling}

Saying that $\left(\nabla_{\boldsymbol{q}} \mathcal{L}, \delta \boldsymbol{q}\right)_{x r}=0$ leads to

$$
\begin{aligned}
- \text { Real }\left(\int_{0}^{L}(\delta \boldsymbol{q},\right. & -E^{+} \frac{\partial \boldsymbol{\lambda}}{\partial x}+\left(A+\alpha B-\frac{\partial E}{\partial x}\right)^{+} \boldsymbol{\lambda}+\frac{\partial \boldsymbol{q}}{\partial x}\left(\zeta-\zeta^{*}\right)-\boldsymbol{q} \frac{\partial \zeta^{*}}{\partial x} \\
& \left.\left.-2\left|e^{i \int_{0}^{x} \alpha(\xi) \mathrm{d} \xi}\right|^{2}\left(\left(Q^{*} Q D_{\overline{\boldsymbol{q}}}\right)^{+} Q^{*} W_{o}(\mathbb{H}(\boldsymbol{q}, \alpha)-\mathcal{Y})\right)\right)_{r} \mathrm{~d} x\right)=0
\end{aligned}
$$

and

$$
-\operatorname{Real}\left(\left[\left(\delta \boldsymbol{q}, E^{+} \boldsymbol{\lambda}\right)_{r}\right]_{0}^{L}\right)+\operatorname{Real}\left(\left(\delta \boldsymbol{q}_{L}, 2\left|e^{i \int_{0}^{L} \alpha(\xi) \mathrm{d} \xi}\right|^{2}\left(\left(Q^{*} Q D_{\overline{\boldsymbol{q}}}\right)^{+} Q^{*} W_{T}\left(\mathbb{H}\left(\boldsymbol{q}_{L}, \alpha_{L}\right)-\mathcal{Y}_{L}\right)\right)\right)_{r}\right)=0 .
$$

Saying that $\left(\nabla_{\alpha} \mathcal{L}, \delta \alpha\right)_{x}=0$ leads to

Real $\left(\int_{0}^{L} \delta \alpha\left(-2 i \int_{x}^{L}(\mathbb{H}(\boldsymbol{q}, \alpha), \mathbb{H}(\alpha)-\mathcal{Y})_{W_{o}} \mathrm{~d} \xi-2 i\left(\mathbb{H}\left(\boldsymbol{q}_{L}, \alpha_{L}\right), \mathbb{H}\left(\alpha_{L}\right)-\mathcal{Y}_{L}\right)_{W_{T}}-(B \boldsymbol{q}, \boldsymbol{\lambda})_{r}\right) \mathrm{d} x\right)=0$. 
If this is true for any $\delta \boldsymbol{q}$ and $\delta \alpha$, and (51) is respected for any $\delta \boldsymbol{w}$, then we get the adjoint system (30) with

$$
\begin{aligned}
& R H S_{1}=2\left|e^{i \int_{0}^{x} \alpha(\xi) \mathrm{d} \xi}\right|^{2}\left(\left(Q^{*} Q D_{\overline{\boldsymbol{q}}}\right)^{+} Q^{*} W_{o}(\mathbb{H}(\boldsymbol{q}, \alpha)-\mathcal{Y})\right) \\
& R H S_{2}=-2 i \int_{x}^{L}(\mathbb{H}(\boldsymbol{q}, \alpha), \mathbb{H}(\boldsymbol{q}, \alpha)-\mathcal{Y})_{W_{o}} \mathrm{~d} \xi-2 i\left(\mathbb{H}\left(\boldsymbol{q}_{L}, \alpha_{L}\right), \mathbb{H}\left(\boldsymbol{q}_{L}, \alpha_{L}\right)-\mathcal{Y}_{L}\right)_{W_{T}} \\
& R H S_{3}=2\left|e^{i \int_{0}^{L} \alpha(\xi) \mathrm{d} \xi}\right|^{2}\left(\left(Q^{*} Q D_{\overline{\boldsymbol{q}}}\right)^{+} Q^{*} W_{T}\left(\mathbb{H}\left(\boldsymbol{q}_{L}, \alpha_{L}\right)-\mathcal{Y}_{L}\right)\right),
\end{aligned}
$$

with $D_{\overline{\boldsymbol{q}}}=\operatorname{diag}(\overline{\boldsymbol{q}})$.

\section{Adjoint of a cumulative integral}

In this section, the adjoint of a cumulative integral is detailed. Let the following term be

$$
\left(\int_{0}^{x} \alpha(\xi) \mathrm{d} \xi, y\right)_{x}=\int_{0}^{L} \int_{0}^{x} \alpha(\xi) \mathrm{d} \xi y(x) \mathrm{d} x .
$$

We define $\beta(x)=\int_{0}^{x} \alpha(\xi) \mathrm{d} \xi$ such that $\frac{\mathrm{d} \beta}{\mathrm{d} x}(x)=\alpha(x)$ and $\gamma(x)=\int_{L}^{x} y(\xi) \mathrm{d} \xi$ such that $\frac{\mathrm{d} \gamma}{\mathrm{d} x}(x)=y(x)$. We have then

$$
\begin{aligned}
\left(\int_{0}^{x} \alpha(\xi) \mathrm{d} \xi, y\right)_{x} & =\int_{0}^{L} \beta(x) \frac{\mathrm{d} \gamma}{\mathrm{d} x}(x) \mathrm{d} x \\
& =[\beta(x) \gamma(x)]_{0}^{L}-\int_{0}^{L} \frac{\mathrm{d} \beta}{\mathrm{d} x}(x) \gamma(x) \mathrm{d} x \\
& =\left[\int_{0}^{x} \alpha(\xi) \mathrm{d} \xi \int_{L}^{x} y(\xi) \mathrm{d} \xi\right]_{0}^{L}-\int_{0}^{L} \alpha(x) \int_{L}^{x} y(\xi) \mathrm{d} \xi \mathrm{d} x \\
& =\left(\alpha(x), \int_{x}^{L} y(\xi) \mathrm{d} \xi\right)_{x}
\end{aligned}
$$

